\title{
An Evaluation of Harmonic Vibrational Frequency Scale
}

\section{Factors}

\author{
Jeffrey P. Merrick, Damian Moran*** and Leo Radom* \\ School of Chemistry and Centre of Excellence in Free Radical Chemistry and \\ Biotechnology, University of Sydney, Sydney, NSW 2006, Australia
}

SUPPORTING INFORMATION

(A total of 22 pages)

*dmoran@cbms.mq.edu.au; radom@chem.usyd.edu.au

$\$$ Current address: Department of Chemistry and Biomolecular Sciences,

Macquarie University, NSW 2109, Australia 
TABLE S1: The F1 Set of Molecules and Their Experimental Frequencies ${ }^{a}$

$\mathrm{AlCl}_{3}: 150,150,183,375,595,595$

$\mathrm{BF}_{3}: 482,482,719,888,1503,1503$

$\mathrm{B}_{2} \mathrm{H}_{6}: 368,816,833,850,930,955,977,1012,1181,1186,1606,1768,1920,2110,2528,2537,2625,2640$

$\mathrm{NClF}_{2}: 377,382,556,697,855,930$

$\mathrm{ClF}_{3}: 328,328,442,529,702,752$

HOCl: $725,1242,3609$

$\mathrm{SiH}_{3} \mathrm{Cl}: 551,664,664,949,949,954,2195,2201,2201$

CINO: $332,596,1800$

$\mathrm{ClNO}_{2}: 370,408,652,793,1286,1685$

CINS: $273,414,1325$

$\mathrm{NCl}_{2} \mathrm{~F}: 274,344,409,615,692,825$

$\mathrm{SiH}_{2} \mathrm{Cl}_{2}: 188,527,590,602,710,876,954,2224,2237$

$\mathrm{Cl}_{2} \mathrm{O}: 296,639,686$

$\mathrm{SOCl}_{2}: 194,284,344,455,492,1251$

$\mathrm{SCl}_{2}: 208,525,535$

$\mathrm{SiHCl}_{3}: 176,176,254,499,600,600,811,811,2261$

$\mathrm{PCl}_{3}: 198,198,252,482,482,504$

HOF: $886,1393,3537$

$\mathrm{SiH}_{3} \mathrm{~F}:$ 728, 728, 872, 956, 956, 990, 2196, 2206, 2206

ONF: $520,766,1844$

NSF: $366,640,1372$

$\mathrm{F}_{2} \mathrm{NH}: 500,888,972,1307,1424,3193$

$\mathrm{N}_{2} \mathrm{~F}_{2}: 364,423,603,991,1018,1523$

$\mathrm{F}_{2} \mathrm{O}: 461,831,928$

$\mathrm{F}_{2} \mathrm{SO}: 378,393,530,747,808,1333$

$\mathrm{S}_{2} \mathrm{~F}_{2}: 183,301,320,615,681,717$

$\mathrm{SiHF}_{3}$ : 306, 306, 425, 844, 858, 858, 998, 998, 2316

$\mathrm{NF}_{3}: 492,492,647,907,907,1032$

$\mathrm{PF}_{3}: 344,344,487,860,860,892$

$\mathrm{HNO}_{3}: 456,579,647,762,879,1325,1331,1708,3550$

$\mathrm{HN}_{3}: 534,607,1151,1264,2140,3336$

$\mathrm{H}_{2} \mathrm{O}: 1595,3657,3756$

$\mathrm{H}_{2} \mathrm{O}_{2}: 371,877,1266,1402,3599,3608$

$\mathrm{H}_{2} \mathrm{~S}: 1183,2615,2626$

$\mathrm{H}_{2} \mathrm{~S}_{2}: 416,509,883,886,2556,2559$

$\mathrm{NH}_{3}: 950,1627,1627,3337,3444,3444$

$\mathrm{PH}_{3}: 992,1118,1118,2323,2328,2328$

$\mathrm{SiH}_{4}:$ 914, 914, 914, 975, 975, 2187, 2191, 2191, 2191

$\mathrm{NO}_{2}: 750,1318,1618$

NNO: 589, 589, 1285, 2224

$\mathrm{SO}_{2}: 518,1151,1362$

$\mathrm{O}_{3}: 701,1042,1103$

$\mathrm{SO}_{3}: 498,530,530,1065,1391,1391$

COClF: 415, 501, 667, 776, 1095, 1868

$\mathrm{ClCN}: 378,378,744,2216$

$\mathrm{COCl}_{2}: 285,440,567,580,849,1827$

$\mathrm{CSCl}_{2}: 220,294,473,505,816,1137$

FCN: 451, 451, 1077, 2323

$\mathrm{COF}_{2}: 584,626,774,965,1249,1928$ 
$\mathrm{CSF}_{2}: 417,526,622,787,1189,1368$

COS: 520, 520, 859, 2062

$\mathrm{CO}_{2}: 667,667,1333,2349$

$\mathrm{CS}_{2}: 397,397,658,1535$

$\mathrm{CHCl}_{3}: 261,261,363,680,774,774,1220,1220,3034$

$\mathrm{CHF}_{3}: 507,507,700,1117,1152,1152,1372,1372,3036$

HCN: 712, 712, 2097, 3311

HNCO: $610,643,762,1327,2274,3531$

$\mathrm{CH}_{2} \mathrm{Cl}_{2}: 282,717,758,898,1153,1268,1467,2999,3040$

$\mathrm{H}_{2} \mathrm{CO}: 1167,1249,1500,1746,2783,2843$

HCOOH: 625, 638, 1033, 1105, 1229, 1387, 1770, 2943, 3570

$\mathrm{BH}_{3} \mathrm{CO}: 314,314,707,819,819,1083,1115,1115,2166,2387,2456,2456$

$\mathrm{CH}_{3} \mathrm{Cl}: 732,1017,1017,1355,1452,1452,2937,3039,3039$

$\mathrm{CH}_{3} \mathrm{~F}: 1049,1182,1182,1464,1467,1467,2930,3006,3006$

$\mathrm{CH}_{4}: 1306,1306,1306,1534,1534,2917,3019,3019,3019$

$\mathrm{CH}_{3} \mathrm{OH}: 250,1033,1060,1165,1345,1455,1477,1477,2844,2960,3000,3681$

$\mathrm{CH}_{3} \mathrm{NH}_{2}: 268,780,1044,1130,1195,1419,1430,1473,1485,1623,2820,2961,2985,3361,3427$

$\mathrm{CH}_{3} \mathrm{SiH}_{3}: 187,540,540,700,868,868,940,980,980,1260,1403,1403,2166,2169,2169,2898,2982,2982$

ClCCCl: $172,172,333,333,477,988,2234$

$\mathrm{C}_{2} \mathrm{~N}_{2}: 234,234,503,503,846,2158,2330$

ClCCH: $326,326,604,604,756,2110,3340$

HCCF: $367,367,578,578,1055,2255,3355$

HCCH: 612, 612, 730, 730, 1974, 3289, 3374

trans $\mathrm{CHCl}=\mathrm{CHCl}: 227,250,350,763,828,846,900,1200,1274,1578,3073,3090$

cis $\mathrm{CHCl}=\mathrm{CHCl}: 173,406,571,697,711,857,876,1179,1303,1587,3072,3077$

$\mathrm{CH}_{2}=\mathrm{CCl}_{2}: 299,372,460,603,686,800,875,1095,1400,1627,3035,3130$

$\mathrm{CHF}=\mathrm{CHF}: 255,482,756,768,866,1014,1127,1266,1376,1715,3135,3135$

trans $\mathrm{CHF}=\mathrm{CHF}: 329,341,548,788,875,1123,1159,1274,1286,1694,3111,3114$

$\mathrm{O}=\mathrm{CHCH}=\mathrm{O}: 127,339,551,801,1048,1065,1312,1338,1732,1745,2835,2843$

$\mathrm{CH}_{3} \mathrm{CN}: 362,362,920,1041,1041,1385,1448,1448,2267,2954,3009,3009$

$\mathrm{CH}_{3} \mathrm{NC}: 263,263,945,1129,1129,1429,1467,1467,2166,2966,3014,3014$

$\mathrm{CH}_{3} \mathrm{COF}: 123,420,567,598,826,1000,1054,1188,1378,1437,1440,1870,2955,3004,3043$

$\mathrm{CH}_{2}=\mathrm{CH}_{2}: 825,943,949,1023,1236,1342,1444,1623,2989,3026,3103,3106$

trans $\mathrm{CH}_{2} \mathrm{ClCH}_{2} \mathrm{Cl}: 123,222,300,728,754,773,989,1052,1123,1232,1264,1304,1445,1461,2957,2983$, 3005,3005

gauche $\mathrm{CH}_{2} \mathrm{ClCH}_{2} \mathrm{Cl}: 125,272,410,669,693,890,948,1027,1146,1207,1292,1315,1433,1436,2957,2957$, 3005,3005

$c-\mathrm{C}_{2} \mathrm{H}_{4} \mathrm{O}: 822,860,877,892,1120,1142,1151,1271,1300,1472,1498,3006,3006,3063,3065$

$\mathrm{CH}_{3} \mathrm{CHO}: 150,509,763,867,919,1113,1352,1400,1420,1441,1743,2822,2917,2967,3005$

$\mathrm{HCOOCH}_{3}: 130,318,332,767,925,1032,1166,1168,1207,1371,1443,1445,1454,1754,2943,2969,3012$, 3045

$\mathrm{CH}_{3} \mathrm{COOH}: 93,534,581,642,657,847,989,1048,1182,1264,1382,1430,1430,1788,2944,2996,3051,3583$

$\mathrm{SiH}_{3} \mathrm{CCH}: 220,220,659,659,668,685,685,935,946,946,2055,2192,2193,2193,3311$

$\mathrm{CH}_{3} \mathrm{CH}_{2} \mathrm{Cl}: 251,336,677,786,974,974,1081,1251,1289,1385,1448,1448,1463,2881,2946,2967,2986,3014$

$\mathrm{CH}_{3} \mathrm{CH}_{2} \mathrm{~F}: 243,415,810,880,1048,1048,1108,1277,1365,1395,1449,1449,1479,2915,2941,3003,3003$, 3003

$c-\mathrm{CH}_{2} \mathrm{CH}_{2} \mathrm{NH}: 773,817,856,904,998,1090,1095,1131,1211,1237,1268,1463,1482,3015,3015,3079,3079$, 3338

$\mathrm{CH}_{3} \mathrm{CH}_{3}: 289,822,822,995,1190,1190,1379,1388,1468,1468,1469,1469,2896,2954,2969,2969,2985,2985$

$\mathrm{CH}_{3} \mathrm{NNCH}_{3}: 222,223,312,352,589,916,1009,1010,1109,1176,1300,1380,1393,1434,1438,1445,1447$, $1580,2916,2926,2966,2977,2982,2982$

$\mathrm{CH}_{3} \mathrm{OCH}_{3}: 203,242,418,928,1102,1150,1179,1227,1244,1452,1452,1464,1464,1464,1464,2817,2817$, 2925, 2952, 2996, 2996 
$\mathrm{CH}_{2}=\mathrm{C}=\mathrm{CHCl}: 184,494,548,592,767,822,875,999,1101,1256,1435,1963,3009,3079,3079$

$\mathrm{HCCCH}_{2} \mathrm{Cl}: 186,311,451,637,650,725,908,960,1179,1271,1441,2147,2968,3002,3335$

$\mathrm{HCCCH}_{2} \mathrm{~F}: 211,310,539,635,675,940,1018,1039,1242,1381,1465,2150,2955,2972,3338$

$\mathrm{CH}_{2}=\mathrm{C}=\mathrm{CH}_{2}: 355,355,841,841,865,999,999,1073,1398,1443,1957,3007,3015,3086,3086$

$\mathrm{CH}_{3} \mathrm{CCH}: 328,328,633,633,931,1053,1053,1382,1452,1452,2142,2918,3008,3008,3334$

$\mathrm{CH}_{2} \mathrm{CHCHO}: 157,327,564,593,912,959,980,993,1158,1275,1360,1420,1625,1724,2800,3000,3028,3103$

$\mathrm{CH}_{3} \mathrm{CH}_{2} \mathrm{CN}: 222,226,378,545,786,836,1005,1022,1077,1256,1319,1387,1433,1465,1465,2254,2849$, 2900, 2995, 3001, 3001

$c-\mathrm{C}_{3} \mathrm{H}_{6}: 739,739,854,866,866,1029,1029,1070,1126,1188,1188,1188,1438,1438,1479,3025,3025,3038$, $3082,3082,3103$

$\mathrm{CH}_{3} \mathrm{COCH}_{3}: 105,109,385,484,530,777,877,891,1066,1091,1216,1364,1364,1410,1426,1435,1454,1731$, 2937, 2937, 2963, 2972, 3019, 3019

HCCCCH: 231, 231, 482, 482, 627, 627, 630, 630, 874, 2020, 2184, 3293, 3329

$\mathrm{CH}_{2}=\mathrm{CHCH}=\mathrm{CH}_{2}: 162,301,512,522,770,894,908,912,976,990,1013,1196,1280,1294,1381,1438,1596$, $1630,2984,2992,3003,3055,3087,3101$

$\mathrm{CH}_{3} \mathrm{CCCH}_{3}: 1,213,213,371,371,725,1029,1029,1054,1054,1152,1380,1382,1448,1448,1456,1456,2240$, 2916, 2938, 2966, 2966, 2973, 2973

$\mathrm{H}_{2}: 4161$

$\mathrm{CH}: 2733$

NH: 3126

$\mathrm{OH}: 3570$

FH: 3961

$\mathrm{SiH}: 1971$

PH: 2276

SH: 2592

$\mathrm{N}_{2}: 2330$

$\mathrm{O}_{2}: 1556$

$\mathrm{F}_{2}: 894$

LiF: 894

$\mathrm{CH}_{2}$ singlet: 1398, 3026, 3074

$\mathrm{CH}_{2}$ triplet: 1056, 2954, 3123

HCO: 1083, 1820, 2488

${ }^{a}$ From (a) Shimanouchi, T. J. Phys. Chem. Ref. Data 1977, 6, 993. (b) Shimanouchi, T. Tables of Molecular

Vibrational Frequencies; NSRDS-NBS 39, National Bureau of Standards: Washington, DC, 1972. 
TABLE S2: The Z1 and Z2 Sets of Molecules and Their Experimental ZPVEs $\left(\mathrm{kJ} \mathrm{mol}^{-1}\right)^{a}$

\begin{tabular}{|c|c|c|c|}
\hline Molecule & ZPVE & Molecule & ZPVE \\
\hline $\mathrm{H}_{2}$ & 25.98 & $\mathrm{SC}$ & 7.66 \\
\hline $\mathrm{CH}$ & 16.90 & $\mathrm{P}_{2}$ & 4.64 \\
\hline $\mathrm{NH}$ & 19.41 & $\mathrm{Cl}_{2}$ & 3.35 \\
\hline $\mathrm{OH}$ & 22.09 & $\mathrm{ClO}$ & 5.10 \\
\hline FH & 24.48 & $\mathrm{ClF}$ & 4.69 \\
\hline $\mathrm{SiH}$ & 12.09 & $\mathrm{CO}_{2}$ & $30.29^{b}$ \\
\hline $\mathrm{PH}$ & 14.02 & $\mathrm{HCN}$ & $41.63^{b}$ \\
\hline $\mathrm{SH}$ & 16.02 & $\mathrm{HCCH}$ & $68.87^{b}$ \\
\hline $\mathrm{N}_{2}$ & 14.06 & $\mathrm{H}_{2} \mathrm{CO}$ & $69.16^{c}$ \\
\hline $\mathrm{O}_{2}$ & 9.41 & $\mathrm{H}_{2} \mathrm{O}$ & $55.44^{c}$ \\
\hline $\mathrm{F}_{2}$ & 5.44 & $\mathrm{H}_{2} \mathrm{~S}$ & $39.33^{c}$ \\
\hline $\mathrm{LiF}$ & 5.44 & $\mathrm{CH}_{2}=\mathrm{CH}_{2}$ & $131.67^{c}$ \\
\hline $\mathrm{Li}_{2}$ & 2.09 & $\mathrm{HCO}$ & $34.14^{c}$ \\
\hline $\mathrm{LiH}$ & 8.33 & $\mathrm{CH}_{4}$ & $115.94^{d}$ \\
\hline $\mathrm{ClH}$ & 17.74 & $\mathrm{CH}_{3} \mathrm{Cl}$ & $98.11^{e}$ \\
\hline BeH & 12.22 & $\mathrm{CH}_{3}$ & $77.61^{f}$ \\
\hline $\mathrm{CN}$ & 12.34 & $\mathrm{NH}_{3}$ & $89.24^{f}$ \\
\hline $\mathrm{CO}$ & 12.93 & $\mathrm{~N}_{2} \mathrm{O}$ & $28.49^{f}$ \\
\hline NO & 11.34 & $\mathrm{NO}_{2}$ & $22.59^{f}$ \\
\hline $\mathrm{Na}_{2}$ & 0.96 & $\mathrm{HOCl}$ & $34.23^{f}$ \\
\hline $\mathrm{NaCl}$ & 2.18 & $\mathrm{SO}_{2}$ & $18.33^{f}$ \\
\hline $\mathrm{SiO}$ & 7.41 & OCS & $23.93^{f}$ \\
\hline $\mathrm{S}_{2}$ & 4.35 & $\mathrm{ClCN}$ & $22.30^{f}$ \\
\hline SO & 6.86 & HNO & $35.82^{f}$ \\
\hline
\end{tabular}

${ }^{a}$ From Huber, K. P.; Herzberg, G. Molecular Spectra and Molecular Structure. IV. Constants of Diatomic

Molecules; Van Nostrand Reinhold: New York, 1979, unless otherwise noted. Z1 set unless otherwise noted. ${ }^{b}$ From Allen, W. D.; Yamaguchi, Y.; Csaszar, A. G.; Clabo, D. A.; Remington, R. B.; Schaefer, H. F. Chem. Phys. 1990, 145, 427. ' From Clabo, D. A.; Allen, W. D.; Remington, R. B.; Yamaguchi, Y.; Schaefer, H. F. Chem. Phys. 1988, 123, 187. ${ }^{d}$ From ref. (Martin, J. M. L.; Lee, T. J.; Taylor, P. R.; Francois, J. P. J. Chem. Phys. 1995, 103, 2589.

${ }^{e}$ From Duncan, J. L.; Law, M. M. J. Mol. Spectrosc. 1990, 140, 13. ${ }^{f}$ Additional molecules in the Z2 set, as listed in Karton, A.; Rabinovich, E.; Martin, J. M. L.; Ruscic, B. J. Chem. Phys. 2006, 125, 144108. 
TABLE S3: Largest rms $_{\text {mol }}$ Values for Fundamental Vibrational Frequencies for Individual Methods

\begin{tabular}{|c|c|}
\hline Method & Largest $\mathrm{rms}_{\mathrm{mol}}$ values $\left(\mathrm{cm}^{-1}\right)$ \\
\hline $\mathrm{HF}$ & $\mathrm{F}_{2}(216-254), \mathrm{O}_{2}(224-249), \mathrm{O}_{3}(217-239), \mathrm{F}_{2} \mathrm{O}(164-192)$, singlet $\mathrm{CH}_{2}(174-185)$ \\
\hline MP2 & $\begin{array}{l}\mathrm{N}_{2}(233-284), \mathrm{O}_{2}(143-228), \mathrm{H}_{2}(120-200) \text {, singlet } \mathrm{CH}_{2}(116-147), \mathrm{FH}(6-31 \mathrm{G}(\mathrm{d}) 143), \mathrm{HCCH}(6- \\
31 \mathrm{G}(\mathrm{d}) 143)\end{array}$ \\
\hline QCISD & singlet $\mathrm{CH}_{2}(172-202), \mathrm{FH}(6-31 \mathrm{G}(\mathrm{d}) \mathrm{130}), \mathrm{HCCH}(6-31 \mathrm{G}(\mathrm{d}) 116)$ \\
\hline QCISD(T) & $\begin{array}{l}\text { singlet } \mathrm{CH}_{2}(163-193), \mathrm{HCCH}(6-31 \mathrm{G}(\mathrm{d}) \text { and } 6-31+\mathrm{G}(\mathrm{d}, \mathrm{p}) 130-158), \mathrm{F}_{2}(6-311+\mathrm{G}(\mathrm{d}, \mathrm{p}) 153), \mathrm{H}_{2} \\
(6-31+\mathrm{G}(\mathrm{d}, \mathrm{p}) \text { and } 6-311+\mathrm{G}(\mathrm{d}, \mathrm{p}) 103-132), \mathrm{FH}(6-31 \mathrm{G}(\mathrm{d}) 117)\end{array}$ \\
\hline CCSD & singlet $\mathrm{CH}_{2}(175-204), \mathrm{FH}(6-31 \mathrm{G}(\mathrm{d}) \mathrm{133}), \mathrm{HCCH}(6-31 \mathrm{G}(\mathrm{d}) 113)$ \\
\hline $\operatorname{CCSD}(\mathrm{T})$ & $\begin{array}{l}\text { singlet } \mathrm{CH}_{2}(164-194), \mathrm{F}_{2}(6-311+\mathrm{G}(\mathrm{d}, \mathrm{p}) 158), \mathrm{HCCH}(6-31 \mathrm{G}(\mathrm{d}) \text { and } 6-31+\mathrm{G}(\mathrm{d}, \mathrm{p}) 128-156), \mathrm{H}_{2} \\
(6-31+\mathrm{G}(\mathrm{d}, \mathrm{p}) \text { and } 6-311+\mathrm{G}(\mathrm{d}, \mathrm{p}) 99-129), \mathrm{FH}(6-31 \mathrm{G}(\mathrm{d}) 119)\end{array}$ \\
\hline B-B95 & singlet $\mathrm{CH}_{2}(213-242), \mathrm{H}_{2}(163-192), \mathrm{FH}\left(6-31+\mathrm{G}(\mathrm{d}, \mathrm{p})\right.$ 143), triplet $\mathrm{CH}_{2}(103-112)$ \\
\hline B-LYP & $\begin{array}{l}\text { singlet } \mathrm{CH}_{2}(173-230), \mathrm{H}_{2}(182-217), \mathrm{HF}(6-31 \mathrm{G}(\mathrm{d}) 174), \mathrm{SO}_{3}(6-31 \mathrm{G}(\mathrm{d}), 6-31+\mathrm{G}(\mathrm{d}, \mathrm{p}) \text { and } 6- \\
311+\mathrm{G}(\mathrm{d}, \mathrm{p}) 112-123)\end{array}$ \\
\hline B-P86 & singlet $\mathrm{CH}_{2}(182-233), \mathrm{H}_{2}(141-170), \mathrm{HF}(6-31 \mathrm{G}(\mathrm{d}) \mathrm{124})$, triplet $\mathrm{CH}_{2}(111-117)$ \\
\hline G96-LYP & singlet $\mathrm{CH}_{2}(177-231), \mathrm{H}_{2}(175-203), \mathrm{HF}(6-31 \mathrm{G}(\mathrm{d}) 148), \mathrm{F}_{2} \mathrm{SO}(6-311+\mathrm{G}(\mathrm{d}, \mathrm{p}) 117)$ \\
\hline НCTH147 & singlet $\mathrm{CH}_{2}(181-223), \mathrm{H}_{2}(145-182), \mathrm{HF}(6-31 \mathrm{G}(\mathrm{d}) 115)$, triplet $\mathrm{CH}_{2}(103-111)$ \\
\hline НCTH407 & singlet $\mathrm{CH}_{2}(182-221), \mathrm{H}_{2}(125-172), \mathrm{HF}(6-31 \mathrm{G}(\mathrm{d}) 125)$, triplet $\mathrm{CH}_{2}(98-109)$ \\
\hline НСТН93 & singlet $\mathrm{CH}_{2}(186-225), \mathrm{H}_{2}(144-191), \mathrm{HF}(6-31 \mathrm{G}(\mathrm{d}) 123)$, triplet $\mathrm{CH}_{2}(100-109)$ \\
\hline O-LYP & singlet $\mathrm{CH}_{2}(187-226), \mathrm{H}_{2}(154-205), \mathrm{HF}\left(6-31 \mathrm{G}(\mathrm{d})\right.$ 137), triplet $\mathrm{CH}_{2}(103-111)$ \\
\hline PBEPBE & singlet $\mathrm{CH}_{2}(180-228), \mathrm{H}_{2}(134-167), \mathrm{HF}\left(6-31 \mathrm{G}(\mathrm{d})\right.$ 137), triplet $\mathrm{CH}_{2}(114-121)$ \\
\hline TPSSTPSS & singlet $\mathrm{CH}_{2}(185-225), \mathrm{H}_{2}(182-219), \mathrm{OH}(6-31 \mathrm{G}(\mathrm{d}) 177), \mathrm{HF}(6-31 \mathrm{G}(\mathrm{d}) 115)$ \\
\hline VSXC & $\begin{array}{l}\text { singlet } \mathrm{CH}_{2}(172-202), \mathrm{H}_{2}(135-164), \mathrm{HF}(6-31 \mathrm{G}(\mathrm{d}) 110), \mathrm{NF}_{3}(6-31+\mathrm{G}(\mathrm{d}, \mathrm{p}) \text { and } 6-311+\mathrm{G}(\mathrm{d}, \mathrm{p}) \\
103-109)\end{array}$ \\
\hline B1-B95 & $\begin{array}{l}\text { singlet } \mathrm{CH}_{2}(197-217), \mathrm{O}_{3}(153-169), \mathrm{F}_{2}(131-167), \mathrm{H}_{2}(6-31+\mathrm{G}(\mathrm{d}, \mathrm{p}) \text { and } 6-31 \mathrm{G}(2 \mathrm{df}, \mathrm{p}) 111-115) \text {, } \\
\mathrm{HF}(6-31 \mathrm{G}(\mathrm{d}) 108)\end{array}$ \\
\hline B1-LYP & $\begin{array}{l}\text { singlet } \mathrm{CH}_{2}(167-204), \mathrm{F}_{2}(6-31 \mathrm{G}(\mathrm{d}), 6-31+\mathrm{G}(\mathrm{d}, \mathrm{p}), 6-31 \mathrm{G}(2 \mathrm{df}, \mathrm{p}) \text { and } 6-311+\mathrm{G}(2 \mathrm{df}, \mathrm{p}) 111-149), \mathrm{H}_{2} \\
(115-145), \mathrm{HF}(6-31 \mathrm{G}(\mathrm{d}) 140), \mathrm{O}_{3}(100-133)\end{array}$ \\
\hline B3-LYP & $\begin{array}{l}\text { singlet } \mathrm{CH}_{2}(168-207), \mathrm{H}_{2}(119-149), \mathrm{F}_{2}(6-31 \mathrm{G}(\mathrm{d}), 6-31+\mathrm{G}(\mathrm{d}, \mathrm{p}), 6-31 \mathrm{G}(2 \mathrm{df}, \mathrm{p}) \text { and 6- } \\
311+\mathrm{G}(2 \mathrm{df}, \mathrm{p}) 105-143),(6-31 \mathrm{G}(\mathrm{d}) 140), \mathrm{O}_{3}(6-31 \mathrm{G}(\mathrm{d}), 6-31+\mathrm{G}(\mathrm{d}, \mathrm{p}), 6-31 \mathrm{G}(2 \mathrm{df}, \mathrm{p}) \text { and 6- } \\
311+\mathrm{G}(2 \mathrm{df}, \mathrm{p}) 100-120)\end{array}$ \\
\hline B3-P86 & $\begin{array}{l}\text { singlet } \mathrm{CH}_{2}(172-208), \mathrm{F}_{2}(6-31 \mathrm{G}(\mathrm{d}), 6-31+\mathrm{G}(\mathrm{d}, \mathrm{p}), 6-31 \mathrm{G}(2 \mathrm{df}, \mathrm{p}) \text { and } 6-311+\mathrm{G}(2 \mathrm{df}, \mathrm{p}) 127-163), \mathrm{O}_{3} \\
(119-149), \mathrm{H}_{2}(6-31+\mathrm{G}(\mathrm{d}, \mathrm{p}) \text { and } 6-31 \mathrm{G}(2 \mathrm{df}, \mathrm{p}) 112-117), \mathrm{HF}(6-31 \mathrm{G}(\mathrm{d}) 104)\end{array}$ \\
\hline B3-PW91 & $\begin{array}{l}\text { singlet } \mathrm{CH}_{2}(180-208), \mathrm{F}_{2}(6-31 \mathrm{G}(\mathrm{d}), 6-31+\mathrm{G}(\mathrm{d}, \mathrm{p}), 6-31 \mathrm{G}(2 \mathrm{df}, \mathrm{p}) \text { and } 6-311+\mathrm{G}(2 \mathrm{df}, \mathrm{p}) 121-157), \mathrm{O}_{3} \\
(117-147), \mathrm{H}_{2}(95-123), \mathrm{HF}(6-31 \mathrm{G}(\mathrm{d}) 107)\end{array}$ \\
\hline B971 & $\begin{array}{l}\text { singlet } \mathrm{CH}_{2}(178-215), \mathrm{H}_{2}(137-162), \mathrm{F}_{2}(6-31 \mathrm{G}(\mathrm{d}), 6-31+\mathrm{G}(\mathrm{d}, \mathrm{p}), 6-31 \mathrm{G}(2 \mathrm{df}, \mathrm{p}) \text { and } 6- \\
311+\mathrm{G}(2 \mathrm{df}, \mathrm{p}) 120-153), \mathrm{O}_{3}(115-144)\end{array}$ \\
\hline B972 & $\begin{array}{l}\text { singlet } \mathrm{CH}_{2}(175-205), \mathrm{O}_{3}(127-154), \mathrm{F}_{2}(6-31 \mathrm{G}(\mathrm{d}), 6-31+\mathrm{G}(\mathrm{d}, \mathrm{p}), 6-31 \mathrm{G}(2 \mathrm{df}, \mathrm{p}) \text { and } 6- \\
311+\mathrm{G}(2 \mathrm{df}, \mathrm{p}) 118-154), \mathrm{H}_{2}(109-140)\end{array}$ \\
\hline B98 & $\begin{array}{l}\text { singlet } \mathrm{CH}_{2}(179-215), \mathrm{F}_{2}(6-31 \mathrm{G}(\mathrm{d}), 6-31+\mathrm{G}(\mathrm{d}, \mathrm{p}), 6-31 \mathrm{G}(2 \mathrm{df}, \mathrm{p}) \text { and } 6-311+\mathrm{G}(2 \mathrm{df}, \mathrm{p}) 125-160), \mathrm{H}_{2} \\
(134-159), \mathrm{O}_{3}(119-148)\end{array}$ \\
\hline $\mathrm{BB} 1 \mathrm{~K}$ & singlet $\mathrm{CH}_{2}(191-206), \mathrm{O}_{3}(198-201), \mathrm{F}_{2}(159-173), \mathrm{O}_{2}(125-131)$ \\
\hline BHandH & $\mathrm{O}_{3}(246-269), \mathrm{F}_{2}(190-256), \mathrm{O}_{2}(183-200)$, singlet $\mathrm{CH}_{2}(170-194), \mathrm{F}_{2} \mathrm{O}(139-167), \mathrm{NO}_{2}(120-129)$ \\
\hline $\begin{array}{l}\text { BHandH- } \\
\text { LYP }\end{array}$ & $\mathrm{O}_{3}(183-207), \mathrm{F}_{2}(131-195)$, singlet $\mathrm{CH}_{2}(167-182), \mathrm{O}_{2}(122-143)$ \\
\hline BMK & singlet $\mathrm{CH}_{2}(181-228), \mathrm{O}_{3}(190-214), \mathrm{F}_{2}(103-172), \mathrm{LiF}(6-31 \mathrm{G}(\mathrm{d})$ and 6-31G(2df,p) 147-161) \\
\hline EDF1 & singlet $\mathrm{CH}_{2}(187-225), \mathrm{H}_{2}(153-184), \mathrm{FH}\left(6-31 \mathrm{G}(\mathrm{d})\right.$ 135), triplet $\mathrm{CH}_{2}(105-111)$ \\
\hline EDF2 & $\begin{array}{l}\text { singlet } \mathrm{CH}_{2}(177-206), \mathrm{OH}(6-31 \mathrm{G}(\mathrm{d}) 140) \mathrm{F}_{2}(6-31 \mathrm{G}(\mathrm{d}) \text { and } 6-31+\mathrm{G}(\mathrm{d}, \mathrm{p}) 118-139), \mathrm{H}_{2}(6- \\
31+\mathrm{G}(\mathrm{d}, \mathrm{p}) 127), \mathrm{FH}(6-31 \mathrm{G}(\mathrm{d}) 123), \mathrm{O}_{3}(6-31 \mathrm{G}(\mathrm{d}) 120)\end{array}$ \\
\hline
\end{tabular}




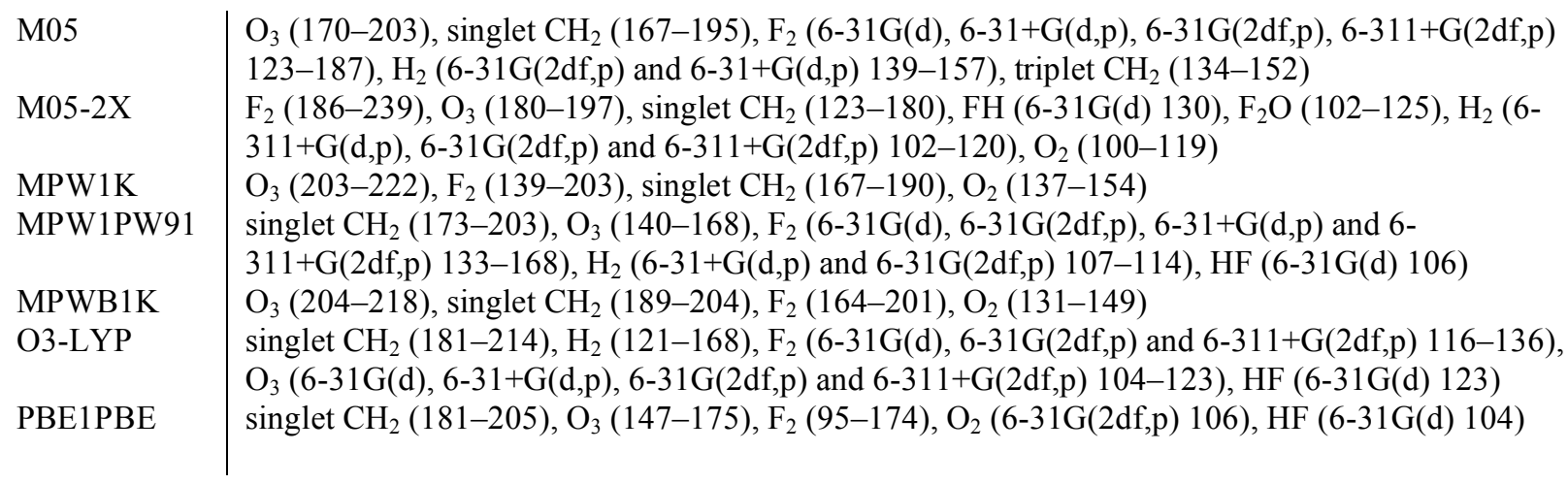




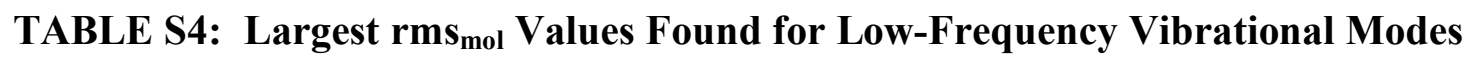

\begin{tabular}{|c|c|}
\hline Method & Largest $\mathrm{rms}_{\mathrm{mol}}$ values $\left(10^{-5} \mathrm{~cm}\right)$ \\
\hline HF & $\begin{array}{l}\mathrm{CH}_{3} \mathrm{NNCH}_{3}(45.6-52.3), \mathrm{ClCCCl}\left(6-31 \mathrm{G}(2 \mathrm{df}, \mathrm{p}) \text { and 6-311+G(2df,p) 44.3-49.2), } \mathrm{CSCl}_{2}(43.4-\right. \\
48.3)\end{array}$ \\
\hline MP2 & $\begin{array}{l}\mathrm{CH}_{3} \mathrm{CCCH}_{3}\left(6-31 \mathrm{G}(\mathrm{d}) \text { and 6-31+G(d,p) 77.2-79.1), } \mathrm{CSCl}_{2}(47.4-59.8), \mathrm{CINS}(6-31 \mathrm{G}(\mathrm{d}), 6-\right. \\
31+\mathrm{G}(\mathrm{d}, \mathrm{p}) \text { and 6-311+G(d,p) 49.2-56.2), } \mathrm{HCCH}(6-31 \mathrm{G}(\mathrm{d}) 53.7)\end{array}$ \\
\hline QCISD & $\begin{array}{l}\mathrm{CSCl}_{2}(53.9-55.9), \mathrm{SiH}_{3} \mathrm{CCH}(6-311+\mathrm{G}(\mathrm{d}, \mathrm{p}) 54.9), \mathrm{CH}_{3} \mathrm{NNCH}_{3}(42.9-53.5), \mathrm{CH}_{3} \mathrm{CCCH}_{3}(6- \\
31 \mathrm{G}(\mathrm{d}) \text { and } 6-31+\mathrm{G}(\mathrm{d}, \mathrm{p}) 43.8-46.2)\end{array}$ \\
\hline QCISD(T) & $\begin{array}{l}\mathrm{SiH}_{3} \mathrm{CCH}(6-311+\mathrm{G}(\mathrm{d}, \mathrm{p}) 89.7), \mathrm{CH}_{3} \mathrm{CCCH}_{3}(6-31 \mathrm{G}(\mathrm{d}) \text { and } 6-31+\mathrm{G}(\mathrm{d}, \mathrm{p}) 71.9-76.3), \mathrm{HCCH}(6- \\
31 \mathrm{G}(\mathrm{d}) \text { and } 6-31+\mathrm{G}(\mathrm{d}, \mathrm{p}) 40.6-68.1), \mathrm{CSCl}_{2}(57.6-60.1), \mathrm{CH}_{3} \mathrm{NNCH}_{3}(47.0-54.9) \text {, }\end{array}$ \\
\hline CCSD & $\begin{array}{l}\mathrm{CSCl}_{2}(53.9-55.7), \mathrm{CH}_{3} \mathrm{NNCH}_{3}(43.5-54.3), \mathrm{SiH}_{3} \mathrm{CCH}(6-311+\mathrm{G}(\mathrm{d}, \mathrm{p}) 53.4), \mathrm{CH}_{3} \mathrm{CCCH}_{3}(6- \\
31 \mathrm{G}(\mathrm{d}) \text { and } 6-31+\mathrm{G}(\mathrm{d}, \mathrm{p}) 43.6-46.2)\end{array}$ \\
\hline $\operatorname{CCSD}(\mathrm{T})$ & $\begin{array}{l}\mathrm{SiH}_{3} \mathrm{CCH}(6-311+\mathrm{G}(\mathrm{d}, \mathrm{p}) 90.5), \mathrm{CH}_{3} \mathrm{CCCH}_{3}(6-31 \mathrm{G}(\mathrm{d}) \text { and } 6-31+\mathrm{G}(\mathrm{d}, \mathrm{p}) 72.2-76.5), \mathrm{HCCH}(6- \\
31 \mathrm{G}(\mathrm{d})(66.5), \mathrm{CSCl}_{2}(57.7-59.9), \mathrm{CH}_{3} \mathrm{NNCH}_{3}(46.9-54.3)\end{array}$ \\
\hline B-B95 & $\begin{array}{l}\mathrm{ClCCCl}(6-31 \mathrm{G}(\mathrm{d}) \text { and } 6-31+\mathrm{G}(\mathrm{d}, \mathrm{p}) 96.5-113.9), \mathrm{CSCl}_{2}(48.1-54.1), \mathrm{SOCl}_{2}(6-31 \mathrm{G}(\mathrm{d}) \text { and } 6- \\
31+\mathrm{G}(\mathrm{d}, \mathrm{p}) 48.5-50.7), \mathrm{ClF}_{3}(48.6)\end{array}$ \\
\hline B-LYP & $\begin{array}{l}\mathrm{ClCCCl}\left(6-31 \mathrm{G}(\mathrm{d}), 6-31+\mathrm{G}(\mathrm{d}, \mathrm{p}) \text { and 6-311+G(d,p) 94.5-143.5), } \mathrm{ClF}_{3}(6-31 \mathrm{G}(\mathrm{d}), 6-31+\mathrm{G}(\mathrm{d}, \mathrm{p}), 6-\right. \\
311+\mathrm{G}(\mathrm{d}, \mathrm{p}) \text { and } 6-311+\mathrm{G}(2 \mathrm{df}, \mathrm{p}) 42.6-77.1), \mathrm{CSCl}_{2}(49.4-57.3), \mathrm{SOCl}_{2}(6-31 \mathrm{G}(\mathrm{d}), 6-31+\mathrm{G}(\mathrm{d}, \mathrm{p}) \\
\text { and } 6-311+\mathrm{G}(\mathrm{d}, \mathrm{p}) 49.3-54.3)\end{array}$ \\
\hline BMK & $\mathrm{CH}_{3} \mathrm{COF}(72.8-78.6), \mathrm{ClCCCl}(38.6-71.1), \mathrm{HCOOCH}_{3}(41.0-59.8), \mathrm{CSCl}_{2}(40.0-46.0)$ \\
\hline B-P86 & $\begin{array}{l}\mathrm{ClCCCl}\left(6-31 \mathrm{G}(\mathrm{d}), 6-31+\mathrm{G}(\mathrm{d}, \mathrm{p}) \text { and } 6-311+\mathrm{G}(\mathrm{d}, \mathrm{p}) \mathrm{101.5-158.4)}, \mathrm{HCOOCH}_{3}(6-31 \mathrm{G}(2 \mathrm{df}, \mathrm{p}) \text { 63.6), }\right. \\
\mathrm{CSCl}_{2}(49.9-59.1), \mathrm{ClF}_{3}(6-31+\mathrm{G}(\mathrm{d}, \mathrm{p}) \text { and 6-311+G(d,p) 44.2-55.7) }\end{array}$ \\
\hline G96-LYP & $\begin{array}{l}\mathrm{ClCCCl}\left(6-31 \mathrm{G}(\mathrm{d}), 6-31+\mathrm{G}(\mathrm{d}, \mathrm{p}) \text { and 6-311+G(d,p) 93.6-136.1), } \mathrm{ClF}_{3}(6-31 \mathrm{G}(\mathrm{d}), 6-31+\mathrm{G}(\mathrm{d}, \mathrm{p}), 6-\right. \\
311+\mathrm{G}(\mathrm{d}, \mathrm{p}) \text { and 6-311+G(2df,p) 39.8-72.6), } \mathrm{HCOOCH}_{3}(6-31 \mathrm{G}(2 \mathrm{df}, \mathrm{p}) 67.3), \mathrm{CSCl}_{2}(49.5-56.7) \\
\mathrm{SOCl}_{2}(6-31 \mathrm{G}(\mathrm{d}), 6-31+\mathrm{G}(\mathrm{d}, \mathrm{p}) \text { and 6-311+G(d,p) 46.6-53.4) }\end{array}$ \\
\hline НCTH147 & $\begin{array}{l}\mathrm{ClCCCl}(6-31 \mathrm{G}(\mathrm{d}), 6-31+\mathrm{G}(\mathrm{d}, \mathrm{p}) \text { and } 6-311+\mathrm{G}(\mathrm{d}, \mathrm{p}) 87.5-136.7), \mathrm{CSCl}_{2}(50.6-58.0), \mathrm{ClF}_{3}(6- \\
311+\mathrm{G}(\mathrm{d}, \mathrm{p}) 49.9)\end{array}$ \\
\hline НCTH407 & $\begin{array}{l}\mathrm{ClCCCl}(6-31 \mathrm{G}(\mathrm{d}), 6-31+\mathrm{G}(\mathrm{d}, \mathrm{p}) \text { and } 6-311+\mathrm{G}(\mathrm{d}, \mathrm{p}) 72.0-123.0), \mathrm{CSCl}_{2}(52.0-58.9), \mathrm{ClF}_{3}(6- \\
311+\mathrm{G}(\mathrm{d}, \mathrm{p}) 47.8)\end{array}$ \\
\hline НСТH93 & $\begin{array}{l}\mathrm{ClCCCl}\left(6-31 \mathrm{G}(\mathrm{d}), 6-31+\mathrm{G}(\mathrm{d}, \mathrm{p}) \text { and 6-311+G(d,p) 65.9-108.9), } \mathrm{CH}_{3} \mathrm{COF}(39.1-60.7), \mathrm{ClF}_{3}(6-\right. \\
31+\mathrm{G}(\mathrm{d}, \mathrm{p}) \text { and } 6-311+\mathrm{G}(\mathrm{d}, \mathrm{p}) 44.4-59.0), \mathrm{CSCl}_{2}(46.6-53.1), \mathrm{SOCl}_{2}(6-31 \mathrm{G}(\mathrm{d}), 6-31+\mathrm{G}(\mathrm{d}, \mathrm{p}) \text { and } \\
6-311+\mathrm{G}(\mathrm{d}, \mathrm{p}) 43.3-47.7)\end{array}$ \\
\hline O-LYP & $\begin{array}{l}\mathrm{ClCCCl}\left(6-31 \mathrm{G}(\mathrm{d}), 6-31+\mathrm{G}(\mathrm{d}, \mathrm{p}) \text { and } 6-311+\mathrm{G}(\mathrm{d}, \mathrm{p}) \text { 98.7-137.5), } \mathrm{CSCl}_{2}(50.3-58.7), \mathrm{ClF}_{3}(6-\right. \\
311+\mathrm{G}(\mathrm{d}, \mathrm{p}) 52.3)\end{array}$ \\
\hline PBEPBE & $\begin{array}{l}\mathrm{ClCCCl}(6-31 \mathrm{G}(\mathrm{d}), 6-31+\mathrm{G}(\mathrm{d}, \mathrm{p}) \text { and } 6-311+\mathrm{G}(\mathrm{d}, \mathrm{p}) 92.0-150.3), \mathrm{CSCl}_{2}(59.3-58.7), \mathrm{ClF}_{3}(6- \\
31+\mathrm{G}(\mathrm{d}, \mathrm{p}) \text { and } 6-311+\mathrm{G}(\mathrm{d}, \mathrm{p}) 41.8-53.7)\end{array}$ \\
\hline TPSSTPSS & $\begin{array}{l}\mathrm{ClCCCl}(6-31 \mathrm{G}(\mathrm{d}), 6-31+\mathrm{G}(\mathrm{d}, \mathrm{p}) \text { and } 6-311+\mathrm{G}(\mathrm{d}, \mathrm{p}) 91.5-136.2) \mathrm{CSCl}_{2}(47.5-55.6), \mathrm{ClF}_{3}(6- \\
311+\mathrm{G}(\mathrm{d}, \mathrm{p}) 46.6), \mathrm{SOCl}_{2}(6-31 \mathrm{G}(\mathrm{d}) \text { and } 6-31+\mathrm{G}(\mathrm{d}, \mathrm{p}) \text { 44.9-45.4) }\end{array}$ \\
\hline VSXC & $\begin{array}{l}\mathrm{ClCCCl}(6-31 \mathrm{G}(\mathrm{d}), 6-31+\mathrm{G}(\mathrm{d}, \mathrm{p}) \text { and } 6-311+\mathrm{G}(\mathrm{d}, \mathrm{p}) 72.1-122.8), \mathrm{HCOOCH}_{3}(54.7-76.4), \mathrm{CSCl}_{2} \\
(50.5-55.9), \mathrm{SOCl}_{2}(6-31 \mathrm{G}(\mathrm{d}), 6-31+\mathrm{G}(\mathrm{d}, \mathrm{p}) \text { and } 6-311+\mathrm{G}(\mathrm{d}, \mathrm{p}) 44.3-45.7)\end{array}$ \\
\hline B1-B95 & $\mathrm{CSCl}_{2}(45.6-50.5), \mathrm{HCOOCH}_{3}(6-31 \mathrm{G}(\mathrm{d})$ and $6-31+\mathrm{G}(\mathrm{d}, \mathrm{p}) 44.1-44.7)$ \\
\hline B1-LYP & $\mathrm{CSCl}_{2}(46.8-52.3)$ \\
\hline B3-LYP & $\mathrm{CSCl}_{2}(47.0-53.1), \mathrm{ClCCCl}(6-311+\mathrm{G}(\mathrm{d}, \mathrm{p}) 49.1), \mathrm{CH}_{3} \mathrm{NNCH}_{3}(6-311+\mathrm{G}(2 \mathrm{df}, \mathrm{p}) 40.2)$ \\
\hline B3-P86 & $\mathrm{ClCCCl}(6-311+\mathrm{G}(\mathrm{d}, \mathrm{p}) 55.0), \mathrm{CSCl}_{2}(48.5-53.9)$ \\
\hline B3-PW91 & $\mathrm{CSCl}_{2}(47.7-54.0), \mathrm{ClCCCl}(6-311+\mathrm{G}(\mathrm{d}, \mathrm{p}) 51.6)$ \\
\hline B971 & $\mathrm{ClCCCl}(6-311+\mathrm{G}(\mathrm{d}, \mathrm{p}) 69.3), \mathrm{CSCl}_{2}(46.2-52.6)$ \\
\hline B972 & $\mathrm{CSCl}_{2}(48.2-54.1), \mathrm{ClCCCl}(6-311+\mathrm{G}(\mathrm{d}, \mathrm{p}) 51.5)$ \\
\hline B98 & $\mathrm{ClCCCl}\left(6-31+\mathrm{G}(\mathrm{d}, \mathrm{p})\right.$ and $6-311+\mathrm{G}(\mathrm{d}, \mathrm{p})$ 41.4-62.1), $\mathrm{CSCl}_{2}(46.0-52.1)$ \\
\hline BB1K & $\mathrm{CSCl}_{2}(48.4-49.8), \mathrm{HCOOCH}_{3}(40.8-45.1)$ \\
\hline BHandH & $\mathrm{CSCl}_{2}(45.1-51.2), \mathrm{ClCCCl}(6-311+\mathrm{G}(2 \mathrm{df}, \mathrm{p}) 42.8)$ \\
\hline
\end{tabular}




\begin{tabular}{|c|c|}
\hline $\begin{array}{l}\text { BHandH- } \\
\text { LYP }\end{array}$ & $\begin{array}{l}\mathrm{CSCl}_{2}(45.1-50.1), \mathrm{CH}_{3} \mathrm{NNCH}_{3}(6-31+\mathrm{G}(\mathrm{d}, \mathrm{p}), 6-311+\mathrm{G}(\mathrm{d}, \mathrm{p}) \text { and } 6-311+\mathrm{G}(2 \mathrm{df}, \mathrm{p}) 41.6-45.1) \text {, } \\
\mathrm{ClCCCl}(6-311+\mathrm{G}(2 \mathrm{df}, \mathrm{p}) 41.1)\end{array}$ \\
\hline EDF1 & $\begin{array}{l}\mathrm{ClCCCl}(84.3-134.9), \mathrm{ClF}_{3}\left(6-31+\mathrm{G}(\mathrm{d}, \mathrm{p}) \text { and } 6-311+\mathrm{G}(\mathrm{d}, \mathrm{p}) \text { 46.6-61.8), } \mathrm{CSCl}_{2}(6-31 \mathrm{G}(\mathrm{d}) \text { and 6- }\right. \\
31+\mathrm{G}(\mathrm{d}, \mathrm{p}) 53.8-55.3), \mathrm{SOCl}_{2}(6-31 \mathrm{G}(\mathrm{d}) \text { and } 6-31+\mathrm{G}(\mathrm{d}, \mathrm{p}) 40.5-41.4)\end{array}$ \\
\hline EDF2 & $\mathrm{BH}_{3} \mathrm{CO}(6-311+\mathrm{G}(\mathrm{d}, \mathrm{p}) 75.2), \mathrm{CSCl}_{2}(49.8-54.1), \mathrm{ClCCCl}(6-311+\mathrm{G}(\mathrm{d}, \mathrm{p}) 51.3)$ \\
\hline M05 & $\mathrm{CSCl}_{2}(44.4-52.1), \mathrm{ClCCCl}(6-31 \mathrm{G}(\mathrm{d}), 6-31+\mathrm{G}(\mathrm{d}, \mathrm{p})$ and $6-311+\mathrm{G}(\mathrm{d}, \mathrm{p}) 40.8-48.0), \mathrm{CH}_{3} \mathrm{COF}(40.2)$ \\
\hline M05-2X & $\begin{array}{l}\mathrm{CH}_{3} \mathrm{COF}(54.7-87.1), \mathrm{CH}_{3} \mathrm{CHO}(6-31+\mathrm{G}(\mathrm{d}, \mathrm{p}), 6-31 \mathrm{G}(2 \mathrm{df}, \mathrm{p}), 6-311+\mathrm{G}(\mathrm{d}, \mathrm{p}) \text { and } 6-311+\mathrm{G}(2 \mathrm{df}, \mathrm{p}) \\
\text { 61.4-66.8), } \mathrm{HCOOCH}_{3}(61.3-68.8), \mathrm{CSCl}_{2}(41.4-46.4)\end{array}$ \\
\hline MPW1K & $\begin{array}{l}\mathrm{CSCl}_{2}(46.0-52.2), \mathrm{CH}_{3} \mathrm{NNCH}_{3}(6-31+\mathrm{G}(\mathrm{d}, \mathrm{p}), 6-311+\mathrm{G}(\mathrm{d}, \mathrm{p}) \text { and } 6-311+\mathrm{G}(2 \mathrm{df}, \mathrm{p}) 40.2-43.2) \\
\mathrm{ClCCCl}(6-311+\mathrm{G}(2 \mathrm{df}, \mathrm{p}) 41.4)\end{array}$ \\
\hline MPW1PW91 & $\mathrm{CSCl}_{2}(47.2-53.5), \mathrm{ClCCCl}(6-311+\mathrm{G}(\mathrm{d}, \mathrm{p}) 42.0)$ \\
\hline MPWB1K & $\mathrm{CSCl}_{2}(45.6-49.8), \mathrm{HCOOCH}_{3}(6-31+\mathrm{G}(\mathrm{d}, \mathrm{p}) 44.7), \mathrm{CH}_{3} \mathrm{NNCH}_{3}(6-31+\mathrm{G}(\mathrm{d}, \mathrm{p}) 40.1)$ \\
\hline O3-LYP & $\mathrm{ClCCCl}(6-31 \mathrm{G}(\mathrm{d}), 6-31+\mathrm{G}(\mathrm{d}, \mathrm{p})$ and $6-311+\mathrm{G}(\mathrm{d}, \mathrm{p}) 48.1-80.2), \mathrm{CSCl}_{2}(48.7-55.6)$ \\
\hline PBE1PBE & $\mathrm{CSCl}_{2}(47.0-53.6), \mathrm{ClCCCl}(6-311+\mathrm{G}(\mathrm{d}, \mathrm{p}) 45.3)$ \\
\hline
\end{tabular}


TABLE S5: Largest rms $_{\text {mol }}$ Values Found for ZPVEs

\begin{tabular}{|c|c|}
\hline Method & Largest $\mathrm{rms}_{\mathrm{mol}}$ values $\left(\mathrm{kJ} \mathrm{mol}^{-1}\right)$ \\
\hline HF & $\begin{array}{l}\mathrm{HCCH}(1.77-2.09), \mathrm{CH}_{4}(1.34-1.94), \mathrm{O}_{2}(1.46-1.61), \mathrm{F}_{2}(1.34-1.57), \mathrm{CN}(1.12-1.51), \mathrm{N}_{2}(1.01- \\
1.09)\end{array}$ \\
\hline MP2 & $\begin{array}{l}\mathrm{HCCH}(0.96-3.54), \mathrm{CH}_{4}(6-31 \mathrm{G}(\mathrm{d}) \text { and } 6-31+\mathrm{G}(\mathrm{d}, \mathrm{p}) 1.34-1.79), \mathrm{HCN}(6-31 \mathrm{G}(\mathrm{d}) \text { and } 6-31+\mathrm{G}(\mathrm{d}, \mathrm{p}) \\
1.19-1.59), \mathrm{N}_{2}(1.24-1.51), \mathrm{CO}_{2}(6-31 \mathrm{G}(\mathrm{d}) \text { and } 6-31+\mathrm{G}(\mathrm{d}, \mathrm{p}) 1.07-1.39), \mathrm{CH}_{3} \mathrm{Cl}(6-31 \mathrm{G}(\mathrm{d}) \text { and } 6- \\
31+\mathrm{G}(\mathrm{d}, \mathrm{p}) 1.01-1.37), \mathrm{SO}(6-31 \mathrm{G}(\mathrm{d}), 6-31+\mathrm{G}(\mathrm{d}, \mathrm{p}), 6-31 \mathrm{G}(2 \mathrm{df}, \mathrm{p}) \text { and } 6-311+\mathrm{G}(\mathrm{d}, \mathrm{p}) 1.03-1.32), \mathrm{O}_{2} \\
(6-31 \mathrm{G}(\mathrm{d}) \text { and } 6-31+\mathrm{G}(\mathrm{d}, \mathrm{p}) 1.26-1.27), \mathrm{HF}(6-31 \mathrm{G}(\mathrm{d}) 1.11)\end{array}$ \\
\hline QCISD & $\begin{array}{l}\mathrm{HCCH}(6-31 \mathrm{G}(\mathrm{d}), 6-31+\mathrm{G}(\mathrm{d}, \mathrm{p}) 2.16-2.42), \mathrm{CH}_{4}(6-31 \mathrm{G}(\mathrm{d}) 1.26), \mathrm{CH}_{3} \mathrm{Cl}(6-31 \mathrm{G}(\mathrm{d}) 1.07), \mathrm{CO}_{2}(6- \\
31+\mathrm{G}(\mathrm{d}, \mathrm{p}) 1.04)\end{array}$ \\
\hline QCISD(T) & $\begin{array}{l}\mathrm{HCCH}(1.11-3.53), \mathrm{CH}_{4}(6-31 \mathrm{G}(\mathrm{d}), 6-31+\mathrm{G}(\mathrm{d}, \mathrm{p}) 1.40-1.73), \mathrm{CH}_{3} \mathrm{Cl}(6-31 \mathrm{G}(\mathrm{d}), 6-31+\mathrm{G}(\mathrm{d}, \mathrm{p}) 1.02- \\
1.37), \mathrm{HCN}(6-31+\mathrm{G}(\mathrm{d}, \mathrm{p}) 1.03), \mathrm{CO}_{2}(6-31+\mathrm{G}(\mathrm{d}, \mathrm{p}) 1.29)\end{array}$ \\
\hline CCSD & $\mathrm{HCCH}(6-31 \mathrm{G}(\mathrm{d}), 6-31+\mathrm{G}(\mathrm{d}, \mathrm{p}) 2.11-2.37), \mathrm{CH}_{4}(6-31 \mathrm{G}(\mathrm{d}) 1.17), \mathrm{CH}_{3} \mathrm{Cl}(6-31 \mathrm{G}(\mathrm{d}) 1.01)$ \\
\hline $\operatorname{CCSD}(\mathrm{T})$ & $\begin{array}{l}\mathrm{HCCH}(1.10-3.50), \mathrm{CH}_{4}(6-31 \mathrm{G}(\mathrm{d}), 6-31+\mathrm{G}(\mathrm{d}, \mathrm{p}) 1.34-1.69), \mathrm{CH}_{3} \mathrm{Cl}(6-31 \mathrm{G}(\mathrm{d}) 1.34), \mathrm{CO}_{2}(6- \\
31+\mathrm{G}(\mathrm{d}, \mathrm{p}) 1.16), \mathrm{HCN}(6-31+\mathrm{G}(\mathrm{d}, \mathrm{p}) 1.02)\end{array}$ \\
\hline B-B95 & $\mathrm{CH}_{4}(6-31 \mathrm{G}(\mathrm{d}) 1.43), \mathrm{HCCH}(6-31 \mathrm{G}(\mathrm{d}) 1.00), \mathrm{HCN}(6-31 \mathrm{G}(2 \mathrm{df}, \mathrm{p}) 1.12)$ \\
\hline B-LYP & $\begin{array}{l}\mathrm{CH}_{4}(6-31 \mathrm{G}(\mathrm{d}) 1.48), \mathrm{FH}(6-31 \mathrm{G}(\mathrm{d}) 1.38), \mathrm{H}_{2} \mathrm{O}(6-31 \mathrm{G}(\mathrm{d}) 1.22), \mathrm{HCN}(6-31 \mathrm{G}(2 \mathrm{df}, \mathrm{p}) 1.23), \mathrm{HCCH} \\
(6-31 \mathrm{G}(\mathrm{d}) 1.16), \mathrm{HCO}(6-31 \mathrm{G}(2 \mathrm{df}, \mathrm{p}) 1.02)\end{array}$ \\
\hline BMK & $\begin{array}{l}\mathrm{CH}_{4}(6-31+\mathrm{G}(\mathrm{d}, \mathrm{p}), 6-31 \mathrm{G}(2 \mathrm{df}, \mathrm{p}) \text { and 6-311+G(2df,p) 1.12-1.41), HCCH }(6-311+\mathrm{G}(\mathrm{d}, \mathrm{p}) 1.27) \\
\mathrm{HCN}(6-31 \mathrm{G}(2 \mathrm{df}, \mathrm{p}) 1.32), \mathrm{F}_{2}(6-31 \mathrm{G}(\mathrm{d}), 6-31+\mathrm{G}(\mathrm{d}, \mathrm{p}), 6-31 \mathrm{G}(2 \mathrm{df}, \mathrm{p}) \text { and 6-311+G(2df,p) 1.01- } \\
1.11), \mathrm{CO}_{2}(6-31 \mathrm{G}(2 \mathrm{df}, \mathrm{p}) 1.11)\end{array}$ \\
\hline B-P86 & $\mathrm{CH}_{4}(6-31 \mathrm{G}(\mathrm{d}) 1.21), \mathrm{HCN}(6-31 \mathrm{G}(2 \mathrm{df}, \mathrm{p}) 1.12), \mathrm{FH}(6-31 \mathrm{G}(\mathrm{d}) 1.05)$ \\
\hline G96-LYP & $\begin{array}{l}\mathrm{CH}_{4}(6-31 \mathrm{G}(\mathrm{d}), 1.36), \mathrm{SC}(6-311+\mathrm{G}(\mathrm{d}, \mathrm{p}) 1.34), \mathrm{FH}(6-31 \mathrm{G}(\mathrm{d}) 1.21), \mathrm{HCN}(6-31 \mathrm{G}(2 \mathrm{df}, \mathrm{p}) 1.16) \\
\mathrm{HCCH}(6-31 \mathrm{G}(\mathrm{d}) 1.06), \mathrm{HCO}(6-31 \mathrm{G}(2 \mathrm{df}, \mathrm{p}) 1.02), \mathrm{H}_{2} \mathrm{O}(6-31 \mathrm{G}(\mathrm{d}) 1.00)\end{array}$ \\
\hline НCТН147 & $\mathrm{CH}_{4}(6-31 \mathrm{G}(\mathrm{d}) 1.09), \mathrm{HCCH}(6-31 \mathrm{G}(\mathrm{d}) 1.02)$ \\
\hline НCTH407 & $\mathrm{HCCH}(6-31 \mathrm{G}(\mathrm{d}) 1.11), \mathrm{CH}_{4}(6-31 \mathrm{G}(\mathrm{d}) 1.09), \mathrm{FH}(6-31 \mathrm{G}(\mathrm{d}) 1.03)$, \\
\hline НCTH93 & $\mathrm{CH}_{4}(6-31 \mathrm{G}(\mathrm{d}) 1.07), \mathrm{HCCH}(6-31 \mathrm{G}(\mathrm{d}) 1.00)$ \\
\hline O-LYP & $\mathrm{CH}_{4}(6-31 \mathrm{G}(\mathrm{d}) 1.25), \mathrm{HCCH}(6-31 \mathrm{G}(\mathrm{d}) 1.17), \mathrm{FH}(6-31 \mathrm{G}(\mathrm{d}) 1.11)$ \\
\hline PBEPBE & $\mathrm{CH}_{4}(6-31 \mathrm{G}(\mathrm{d}) 1.17), \mathrm{FH}(6-31 \mathrm{G}(\mathrm{d}) 1.12)$ \\
\hline TPSSTPSS & $\mathrm{FH}(6-31 \mathrm{G}(\mathrm{d}) 1.41), \mathrm{H}_{2} \mathrm{O}(6-31 \mathrm{G}(\mathrm{d}) 1.41), \mathrm{CH}_{4}(6-31 \mathrm{G}(\mathrm{d}) 1.37), \mathrm{OH}(6-31 \mathrm{G}(\mathrm{d}) 1.03)$ \\
\hline VSXC & $\mathrm{CH}_{4}(6-31 \mathrm{G}(\mathrm{d}) 1.42), \mathrm{HCCH}(6-31 \mathrm{G}(\mathrm{d}) 1.13), \mathrm{HCN}(6-31 \mathrm{G}(2 \mathrm{df}, \mathrm{p}) 1.01)$ \\
\hline B1-B95 & $\mathrm{HCN}(6-31 \mathrm{G}(2 \mathrm{df}, \mathrm{p}) 1.27), \mathrm{F}_{2}(6-31 \mathrm{G}(2 \mathrm{df}, \mathrm{p}) 1.05)$ \\
\hline B1-LYP & HCCH (6-311+G(d,p) 1.36), HCN (6-31G(2df,p) 1.35), FH (6-31G(d) 1.15) \\
\hline B3-LYP & $\mathrm{HCCH}(6-311+\mathrm{G}(\mathrm{d}, \mathrm{p}) 1.30), \mathrm{FH}(6-31 \mathrm{G}(\mathrm{d}) 1.15)$ \\
\hline B3-P86 & $\mathrm{HCCH}(6-311+\mathrm{G}(\mathrm{d}, \mathrm{p}) 1.23), \mathrm{HCN}(6-31 \mathrm{G}(2 \mathrm{df}, \mathrm{p}) 1.21), \mathrm{F}_{2}(6-31 \mathrm{G}(2 \mathrm{df}, \mathrm{p}) 1.02)$ \\
\hline B3-PW91 & $\mathrm{HCCH}(6-311+\mathrm{G}(\mathrm{d}, \mathrm{p}) 1.26), \mathrm{HCN}(6-31 \mathrm{G}(2 \mathrm{df}, \mathrm{p}) 1.20)$ \\
\hline B971 & $\mathrm{HCCH}(6-311+\mathrm{G}(\mathrm{d}, \mathrm{p}) 1.12), \mathrm{HCN}(6-31 \mathrm{G}(2 \mathrm{df}, \mathrm{p}) 1.11)$ \\
\hline B972 & $\mathrm{HCCH}(6-311+\mathrm{G}(\mathrm{d}, \mathrm{p}) 1.13), \mathrm{HCN}(6-31 \mathrm{G}(2 \mathrm{df}, \mathrm{p}) 1.12)$ \\
\hline B98 & $\mathrm{HCCH}(6-311+\mathrm{G}(\mathrm{d}, \mathrm{p}) 1.17), \mathrm{HCN}(6-31 \mathrm{G}(2 \mathrm{df}, \mathrm{p}) 1.14)$ \\
\hline $\mathrm{BB} 1 \mathrm{~K}$ & $\mathrm{HCN}(6-31 \mathrm{G}(2 \mathrm{df}, \mathrm{p}) 1.33), \mathrm{F}_{2}(1.01-1.23), \mathrm{HCCH}(6-31 \mathrm{G}(2 \mathrm{df}, \mathrm{p}) 1.16)$ \\
\hline BHandH & $\begin{array}{l}\mathrm{HCCH}(6-31+\mathrm{G}(\mathrm{d}, \mathrm{p}), 6-31 \mathrm{G}(2 \mathrm{df}, \mathrm{p}), 6-311+\mathrm{G}(\mathrm{d}, \mathrm{p}) \text { and } 6-311+\mathrm{G}(2 \mathrm{df}, \mathrm{p}) 1.22-1.74), \mathrm{F}_{2}(1.21-1.61) \text {, } \\
\mathrm{HCN}(1.04-1.54), \mathrm{CH}_{4}(6-31+\mathrm{G}(\mathrm{d}, \mathrm{p}), 6-31 \mathrm{G}(2 \mathrm{df}, \mathrm{p}), 6-311+\mathrm{G}(\mathrm{d}, \mathrm{p}) \text { and } 6-311+\mathrm{G}(2 \mathrm{df}, \mathrm{p}) 1.19-1.45), \\
\mathrm{O}_{2}(1.24-1.36), \mathrm{CO}_{2}(6-31 \mathrm{G}(2 \mathrm{df}, \mathrm{p}), 6-311+\mathrm{G}(\mathrm{d}, \mathrm{p}) \text { and } 6-311+\mathrm{G}(2 \mathrm{df}, \mathrm{p}) 1.15-1.29) \text {, NO (1.06- } \\
1.14), \mathrm{CH}_{3} \mathrm{Cl}(6-31 \mathrm{G}(2 \mathrm{df}, \mathrm{p}) 1.03)\end{array}$ \\
\hline BHandH- & $\mathrm{HCCH}(6-31+\mathrm{G}(\mathrm{d}, \mathrm{p}), 6-31 \mathrm{G}(2 \mathrm{df}, \mathrm{p}), 6-311+\mathrm{G}(\mathrm{d}, \mathrm{p})$ and 6-311+G(2df,p) 1.11-1.67), HCN (6- \\
\hline LYP & $\begin{array}{l}31 \mathrm{G}(\mathrm{d}), 6-31 \mathrm{G}(2 \mathrm{df}, \mathrm{p}), 6-311+\mathrm{G}(\mathrm{d}, \mathrm{p}) \text { and } 6-311+\mathrm{G}(2 \mathrm{df}, \mathrm{p}) 1.03-1.47), \mathrm{F}_{2}(6-31 \mathrm{G}(\mathrm{d}), 6-31+\mathrm{G}(\mathrm{d}, \mathrm{p}) \\
6-31 \mathrm{G}(2 \mathrm{df}, \mathrm{p}) \text { and } 6-311+\mathrm{G}(2 \mathrm{df}, \mathrm{p}) 1.00-1.22), \mathrm{CH}_{4}(6-311+\mathrm{G}(\mathrm{d}, \mathrm{p}) \text { and } 6-311+\mathrm{G}(2 \mathrm{df}, \mathrm{p}) 1.04-1.06)\end{array}$ \\
\hline EDF1 & $\mathrm{CH}_{4}(6-31 \mathrm{G}(\mathrm{d}) 1.20), \mathrm{FH}(6-31 \mathrm{G}(\mathrm{d}) 1.10), \mathrm{HCCH}(6-31 \mathrm{G}(\mathrm{d}) 1.00)$ \\
\hline EDF2 & $\mathrm{HCCH}(6-311+\mathrm{G}(\mathrm{d}, \mathrm{p}) 1.33), \mathrm{FH}(6-31 \mathrm{G}(\mathrm{d}) 1.02)$ \\
\hline M05 & $\mathrm{HCCH}(6-311+\mathrm{G}(\mathrm{d}, \mathrm{p}) 1.19), \mathrm{F}_{2}(6-31 \mathrm{G}(2 \mathrm{df}, \mathrm{p}) 1.18)$ \\
\hline
\end{tabular}


M05-2X $\quad F_{2}(1.16-1.49)$, HCCH (6-31G(2df,p), 6-311+G(d,p) 1.05-1.23), HCN (6-31G(d), 6-31G(2df,p), 6$311+\mathrm{G}(\mathrm{d}, \mathrm{p})$ and $6-311+\mathrm{G}(2 \mathrm{df}, \mathrm{p}) 1.07-1.19)$, FH $(6-31 \mathrm{G}(\mathrm{d}) 1.05)$

MPW1K $\quad \mathrm{HCCH}(6-31 \mathrm{G}(2 \mathrm{df}, \mathrm{p}), 6-311+\mathrm{G}(\mathrm{d}, \mathrm{p})$ and 6-311+G(2df,p) 1.18-1.50), HCN (6-31G(2df,p) and 6$311+\mathrm{G}(\mathrm{d}, \mathrm{p}) 1.01-1.31), \mathrm{F}_{2}(6-31 \mathrm{G}(\mathrm{d}), 6-31 \mathrm{G}(2 \mathrm{df}, \mathrm{p}), 6-311+\mathrm{G}(\mathrm{d}, \mathrm{p})$ and 6-311+G(2df,p) 1.06-1.27), $\mathrm{O}_{2}\left(6-31 \mathrm{G}(2 \mathrm{df}, \mathrm{p})\right.$ and 6-311+G(d,p)1.00-1.05), $\mathrm{CH}_{4}(6-311+\mathrm{G}(\mathrm{d}, \mathrm{p}) 1.03)$

MPW1PW91 HCCH (6-311+G(d,p) 1.31), HCN (6-31G(2df,p) 1.21), F (6-31G(2df,p) 1.06)

MPWB1K

$\mathrm{HCN}(6-31 \mathrm{G}(2 \mathrm{df}, \mathrm{p}) 1.33), \mathrm{F}_{2}(1.04-1.26), \mathrm{HCCH}(6-31 \mathrm{G}(2 \mathrm{df}, \mathrm{p}) 1.14), \mathrm{O}_{2}$ (6-31G(2df,p) 1.03)

O3-LYP $\quad$ FH $(6-31 \mathrm{G}(\mathrm{d}) 1.02)$

PBE1PBE

HCCH (6-311+G(d,p) 1.27), HCN (6-31G(2df,p) 1.23), F (6-31G(2df,p) 1.09) 
TABLE S6: Largest rms $_{\text {mol }}$ Values Found for $\Delta H_{\text {vib }}(298.15 \mathrm{~K})$

\begin{tabular}{|c|c|}
\hline Method & Largest $\mathrm{rms}_{\text {mol }}$ values $\left(\mathrm{kJ} \mathrm{mol}^{-1}\right)$ \\
\hline $\mathrm{HF}$ & $\begin{array}{l}\mathrm{HCCF}(0.12-0.17), \mathrm{HCCCCH}(6-31+\mathrm{G}(\mathrm{d}, \mathrm{p}) \text { and } 6-31 \mathrm{G}(\mathrm{d}) 0.12-0.13), \mathrm{ClNO}(6-311+\mathrm{G}(\mathrm{d}, \mathrm{p}) 0.12) \text {, } \\
\mathrm{ClCCCl}(6-31 \mathrm{G}(\mathrm{d}), 6-31+\mathrm{G}(\mathrm{d}, \mathrm{p}), 6-31 \mathrm{G}(2 \mathrm{df}, \mathrm{p}) \text { and } 6-311+\mathrm{G}(2 \mathrm{df}, \mathrm{p}) 0.11-0.17), \mathrm{ONF}(0.10-0.13) \text {, } \\
\mathrm{CH} 2=\mathrm{C}=\mathrm{CHCl}(0.11-0.12), \mathrm{CSCl}_{2}(6-31 \mathrm{G}(\mathrm{d}), 6-31+\mathrm{G}(\mathrm{d}, \mathrm{p}) \text { and } 6-311+\mathrm{G}(\mathrm{d}, \mathrm{p}) 0.10-0.11), \mathrm{F}_{2} \mathrm{O}(6- \\
31 \mathrm{G}(2 \mathrm{df}, \mathrm{p}), 6-311+\mathrm{G}(\mathrm{d}, \mathrm{p}) \text { and } 6-311+\mathrm{G}(2 \mathrm{df}, \mathrm{p}) 0.11), \mathrm{FCN}(6-311+\mathrm{G}(2 \mathrm{df}, \mathrm{p}) 0.11)\end{array}$ \\
\hline MP2 & $\begin{array}{l}\mathrm{ClCCCl}(0.38-0.40), \mathrm{ClCCH}(0.31-0.34), \mathrm{HCCCCH}(6-311+\mathrm{G}(\mathrm{d}, \mathrm{p}) \text { and 6-31G(d) 0.28-0.32), } \\
\mathrm{HCCH}(6-31 \mathrm{G}(\mathrm{d}) \text { and } 6-31+\mathrm{G}(\mathrm{d}, \mathrm{p}) 0.13-0.24), \mathrm{CH}_{3} \mathrm{CCCH}_{3}(6-31 \mathrm{G}(\mathrm{d}) \text { and } 6-31+\mathrm{G}(\mathrm{d}, \mathrm{p}) 0.19) \\
\mathrm{ClNS}(0.13-0.15), \mathrm{CSCl} \mathrm{Cl}_{2}(0.15), \mathrm{NSF}(6-311+\mathrm{G}(\mathrm{d}, \mathrm{p}) 0.15), \mathrm{CH}_{3} \mathrm{CCH}(6-31 \mathrm{G}(\mathrm{d}) \text { and } 6-31+\mathrm{G}(\mathrm{d}, \mathrm{p}) \\
0.10-0.14), \mathrm{CH} 2=\mathrm{C}=\mathrm{CHCl}(0.11-0.13)\end{array}$ \\
\hline QCISD & $\begin{array}{l}\mathrm{HCCH}(6-31 \mathrm{G}(\mathrm{d}) \text { and } 6-31+\mathrm{G}(\mathrm{d}, \mathrm{p}) 0.13-0.20), \mathrm{CH} 2=\mathrm{C}=\mathrm{CHCl}(0.12-0.14), \mathrm{CSCl}_{2}(0.13) \\
\mathrm{CH}_{3} \mathrm{CCCH}_{3}(6-31 \mathrm{G}(\mathrm{d}) \text { and } 6-31+\mathrm{G}(\mathrm{d}, \mathrm{p}) 0.13)\end{array}$ \\
\hline QCISD(T) & $\begin{array}{l}\mathrm{HCCH}(6-31 \mathrm{G}(\mathrm{d}) \text { and } 6-31+\mathrm{G}(\mathrm{d}, \mathrm{p}) 0.20-0.31), \mathrm{CH}_{3} \mathrm{CCCH}_{3}(0.10-0.19), \mathrm{CH}_{3} \mathrm{CCH}(6-31 \mathrm{G}(\mathrm{d}) \text { and } \\
6-31+\mathrm{G}(\mathrm{d}, \mathrm{p}) 0.13-0.15), \mathrm{CH}=\mathrm{C}=\mathrm{CHCl}(0.12-0.15), \mathrm{CSCl}_{2}(0.14), \mathrm{SiH}_{3} \mathrm{CCH}(6-311+\mathrm{G}(\mathrm{d}, \mathrm{p}) 0.13)\end{array}$ \\
\hline CCSD & $\begin{array}{l}\mathrm{HCCH}(6-31 \mathrm{G}(\mathrm{d}) \text { and } 6-31+\mathrm{G}(\mathrm{d}, \mathrm{p}) 0.12-19), \mathrm{CH} 2=\mathrm{C}=\mathrm{CHCl}(0.12-0.14), \mathrm{CSCl}_{2}(0.13) \\
\mathrm{CH}_{3} \mathrm{CCCH}_{3}(6-31 \mathrm{G}(\mathrm{d}) \text { and } 6-31+\mathrm{G}(\mathrm{d}, \mathrm{p}) 0.13)\end{array}$ \\
\hline $\operatorname{CCSD}(\mathrm{T})$ & $\begin{array}{l}\mathrm{HCCH}(6-31 \mathrm{G}(\mathrm{d}) \text { and } 6-31+\mathrm{G}(\mathrm{d}, \mathrm{p}) 0.20-0.30), \mathrm{CH}_{3} \mathrm{CCCH}_{3}(0.10-0.19), \mathrm{CH} 2=\mathrm{C}=\mathrm{CHCl}(0.12- \\
0.15), \mathrm{CH}_{3} \mathrm{CCH}(6-31 \mathrm{G}(\mathrm{d}) \text { and } 6-31+\mathrm{G}(\mathrm{d}, \mathrm{p}) 0.13-0.15), \mathrm{CSCl}_{2}(0.14), \mathrm{SiH}_{3} \mathrm{CCH}(6-311+\mathrm{G}(\mathrm{d}, \mathrm{p}) \\
0.13)\end{array}$ \\
\hline B-B95 & $\begin{array}{l}\mathrm{ClCCCl}(6-31 \mathrm{G}(\mathrm{d}) \text { and } 6-31+\mathrm{G}(\mathrm{d}, \mathrm{p}) 0.23-0.27), \mathrm{ClF}_{3}(6-31 \mathrm{G}(\mathrm{d}) \text { and } 6-31+\mathrm{G}(\mathrm{d}, \mathrm{p}) 0.12-0.15) \\
\mathrm{HCCH}(6-31 \mathrm{G}(\mathrm{d}) \text { and } 6-31 \mathrm{G}(2 \mathrm{df}, \mathrm{p}) 0.10-0.14), \mathrm{CSCl}_{2}(0.11-0.13), \mathrm{HCCCCH}(6-31+\mathrm{G}(\mathrm{d}, \mathrm{p}) 0.12)\end{array}$ \\
\hline B-LYP & $\begin{array}{l}\mathrm{ClCCCl}(6-31 \mathrm{G}(\mathrm{d}), 6-31+\mathrm{G}(\mathrm{d}, \mathrm{p}) \text { and } 6-311+\mathrm{G}(\mathrm{d}, \mathrm{p}) 0.23-0.31), \mathrm{ClF}_{3}(0.11-0.23), \mathrm{HCCH}(6-31 \mathrm{G}(\mathrm{d}) \\
6-31 \mathrm{G}(2 \mathrm{df}, \mathrm{p}) 0.14-0.16), \mathrm{CSCl}_{2}(0.12-0.14), \mathrm{F}_{2} \mathrm{SO}(6-31+\mathrm{G}(\mathrm{d}, \mathrm{p}) \text { and } 6-311+\mathrm{G}(\mathrm{d}, \mathrm{p}) 0.12-0.14) \text {, } \\
\mathrm{HCCCCH}(6-31 \mathrm{G}(\mathrm{d}) \text { and } 6-31+\mathrm{G}(\mathrm{d}, \mathrm{p}) 0.10-0.13)\end{array}$ \\
\hline B-P86 & $\begin{array}{l}\mathrm{ClCCCl}(6-31 \mathrm{G}(\mathrm{d}), 6-31+\mathrm{G}(\mathrm{d}, \mathrm{p}) \text { and 6-311+G(d,p) } 0.24-0.32), \mathrm{ClF}_{3}(6-31 \mathrm{G}(\mathrm{d}), 6-31+\mathrm{G}(\mathrm{d}, \mathrm{p}), 6- \\
311+\mathrm{G}(\mathrm{d}, \mathrm{p}) \text { and 6-311+G(2df,p) } 0.11-0.18), \mathrm{CSCl} 2(0.12-0.14), \mathrm{HCCH}(6-31 \mathrm{G}(\mathrm{d}) \text { and 6- } \\
31 \mathrm{G}(2 \mathrm{df}, \mathrm{p}) 0.10-0.13), \mathrm{CH} 2=\mathrm{C}=\mathrm{CHCl}(0.11-0.12), \mathrm{ClCCH}(6-31 \mathrm{G}(\mathrm{d}) 0.12), \mathrm{HCCCCH}(6- \\
31+\mathrm{G}(\mathrm{d}, \mathrm{p}) 0.12)\end{array}$ \\
\hline G96-LYP & $\begin{array}{l}\mathrm{ClCCCl}(6-31 \mathrm{G}(\mathrm{d}), 6-31+\mathrm{G}(\mathrm{d}, \mathrm{p}) \text { and } 6-311+\mathrm{G}(\mathrm{d}, \mathrm{p}) 0.23-0.30), \mathrm{ClF}_{3}(0.11-0.22), \mathrm{CSCl}_{2}(0.12- \\
0.14), \mathrm{F}_{2} \mathrm{SO}(6-31+\mathrm{G}(\mathrm{d}, \mathrm{p}) \text { and } 6-311+\mathrm{G}(\mathrm{d}, \mathrm{p}) 0.11-0.14), \mathrm{HCCH}(6-31 \mathrm{G}(\mathrm{d}) \text { and } 6-31+\mathrm{G}(\mathrm{d}, \mathrm{p}) 0.11- \\
0.14), \mathrm{HCCCCH}(6-31 \mathrm{G}(\mathrm{d}) \text { and } 6-31+\mathrm{G}(\mathrm{d}, \mathrm{p}) 0.10-0.13)\end{array}$ \\
\hline НCTH147 & $\begin{array}{l}\mathrm{ClCCCl}(6-31 \mathrm{G}(\mathrm{d}), 6-31+\mathrm{G}(\mathrm{d}, \mathrm{p}) \text { and } 6-311+\mathrm{G}(\mathrm{d}, \mathrm{p}) 0.22-0.30), \mathrm{ClF}_{3}(6-31+\mathrm{G}(\mathrm{d}, \mathrm{p}) \text { and 6- } \\
311+\mathrm{G}(\mathrm{d}, \mathrm{p}) 0.13-0.017), \mathrm{CSCl}_{2}(0.12-0.14), \mathrm{HCCH}(6-31 \mathrm{G}(\mathrm{d}) \text { and } 6-31 \mathrm{G}(2 \mathrm{df}, \mathrm{p}) 0.10-0.13) \\
\mathrm{CH} 2=\mathrm{C}=\mathrm{CHCl}(0.11-0.12)\end{array}$ \\
\hline НCTH407 & $\begin{array}{l}\mathrm{ClCCCl}(6-31 \mathrm{G}(\mathrm{d}), 6-31+\mathrm{G}(\mathrm{d}, \mathrm{p}) \text { and 6-311+G(d,p) } 0.19-0.28), \mathrm{ClF}_{3}(6-31+\mathrm{G}(\mathrm{d}, \mathrm{p}) \text { and 6- } \\
311+\mathrm{G}(\mathrm{d}, \mathrm{p}) 0.12-0.017), \mathrm{CSCl}_{2}(0.12-0.14), \mathrm{HCCH}(6-31 \mathrm{G}(\mathrm{d}) \text { and 6-31G(2df,p) 0.11-0.13), } \\
\mathrm{CH} 2=\mathrm{C}=\mathrm{CHCl}(0.11-0.12)\end{array}$ \\
\hline НСТН93 & $\begin{array}{l}\mathrm{ClCCCl}(6-31 \mathrm{G}(\mathrm{d}), 6-31+\mathrm{G}(\mathrm{d}, \mathrm{p}) \text { and 6-311+G(d,p) } 0.16-0.25), \mathrm{ClF}_{3}(6-31 \mathrm{G}(\mathrm{d}), 6-31+\mathrm{G}(\mathrm{d}, \mathrm{p}), 6- \\
311+\mathrm{G}(\mathrm{d}, \mathrm{p}) \text { and } 6-311+\mathrm{G}(2 \mathrm{df}, \mathrm{p}) 0.10-0.018), \mathrm{CSCl}_{2}(0.12-0.14), \mathrm{HCCH}(6-31 \mathrm{G}(\mathrm{d}) \text { and 6- } \\
31 \mathrm{G}(2 \mathrm{df}, \mathrm{p}) 0.11-0.13), \mathrm{CH}_{3} \mathrm{OH}(6-31 \mathrm{G}(\mathrm{d}), 6-31+\mathrm{G}(\mathrm{d}, \mathrm{p}) \text { and 6-31G(2df,p) } 0.10-0.12),\left(\mathrm{Cl}_{2} \mathrm{O} \text { 6- }\right. \\
311+\mathrm{G}(\mathrm{d}, \mathrm{p}) 0.12), \mathrm{CH} 2=\mathrm{C}=\mathrm{CHCl}(0.11)\end{array}$ \\
\hline O-LYP & $\begin{array}{l}\mathrm{ClCCCl}(6-31 \mathrm{G}(\mathrm{d}), 6-31+\mathrm{G}(\mathrm{d}, \mathrm{p}) \text { and } 6-311+\mathrm{G}(\mathrm{d}, \mathrm{p}) 0.24-0.30), \mathrm{ClF}_{3}(6-31+\mathrm{G}(\mathrm{d}, \mathrm{p}), 6-311+\mathrm{G}(\mathrm{d}, \mathrm{p}) \\
\text { and } 6-311+\mathrm{G}(2 \mathrm{df}, \mathrm{p}) 0.10-0.17), \mathrm{CSCl}_{2}(0.12-0.14), \mathrm{HCCH}(6-31 \mathrm{G}(\mathrm{d}) \text { and } 6-31 \mathrm{G}(2 \mathrm{df}, \mathrm{p}) 0.12- \\
0.14), \mathrm{CH} 2=\mathrm{C}=\mathrm{CHCl}(0.11-0.12), \mathrm{ClCCH}(6-31 \mathrm{G}(\mathrm{d}) 0.12)\end{array}$ \\
\hline PBEPBE & $\begin{array}{l}\mathrm{ClCCCl}(6-31 \mathrm{G}(\mathrm{d}), 6-31+\mathrm{G}(\mathrm{d}, \mathrm{p}) \text { and } 6-311+\mathrm{G}(\mathrm{d}, \mathrm{p}) 0.23-0.31), \mathrm{ClF}_{3}(6-31 \mathrm{G}(\mathrm{d}), 6-31+\mathrm{G}(\mathrm{d}, \mathrm{p}), 6- \\
311+\mathrm{G}(\mathrm{d}, \mathrm{p}) \text { and 6-311+G(2df,p) } 0.10-0.18), \mathrm{CSCl}_{2}(0.12-0.14), \mathrm{HCCH}(6-31 \mathrm{G}(\mathrm{d}) \text { and 6- } \\
31 \mathrm{G}(2 \mathrm{df}, \mathrm{p}) 0.10-0.13), \mathrm{CH} 2=\mathrm{C}=\mathrm{CHCl}(0.11-0.12), \mathrm{F}_{2} \mathrm{SO}(6-31+\mathrm{G}(\mathrm{d}, \mathrm{p}) \text { and } 6-311+\mathrm{G}(\mathrm{d}, \mathrm{p}) 0.10- \\
0.12) \text {, HCCCCH }(6-31+\mathrm{G}(\mathrm{d}, \mathrm{p}) 0.12) \text {, ClCCH }(6-31 \mathrm{G}(\mathrm{d}) 0.11)\end{array}$ \\
\hline TPSSTPSS & $\begin{array}{l}\mathrm{ClCCCl}(6-31 \mathrm{G}(\mathrm{d}), 6-31+\mathrm{G}(\mathrm{d}, \mathrm{p}) \text { and } 6-311+\mathrm{G}(\mathrm{d}, \mathrm{p}) 0.22-0.30), \mathrm{ClF}_{3}(6-31+\mathrm{G}(\mathrm{d}, \mathrm{p}) \text { and } 6- \\
311+\mathrm{G}(\mathrm{d}, \mathrm{p}) 0.13-0.16), \mathrm{CSCl}_{2}(0.11-0.13), \mathrm{HCCCCH}(6-31+\mathrm{G}(\mathrm{d}, \mathrm{p}) 0.13), \mathrm{F}_{2} \mathrm{SO}(6-31+\mathrm{G}(\mathrm{d}, \mathrm{p}) \text { and }\end{array}$ \\
\hline
\end{tabular}




\begin{tabular}{|c|c|}
\hline & $6-311+\mathrm{G}(\mathrm{d}, \mathrm{p})$ 0.10-0.11), $\mathrm{CH} 2=\mathrm{C}=\mathrm{CHCl}(0.11)$ \\
\hline VSXC & $\begin{array}{l}\mathrm{ClCCCl}(6-31 \mathrm{G}(\mathrm{d}), 6-31+\mathrm{G}(\mathrm{d}, \mathrm{p}) \text { and } 6-311+\mathrm{G}(\mathrm{d}, \mathrm{p}) 0.18-0.28), \mathrm{ClF}_{3}(6-31+\mathrm{G}(\mathrm{d}, \mathrm{p}) \text { and } 6- \\
311+\mathrm{G}(\mathrm{d}, \mathrm{p}) 0.11-0.15), \mathrm{CSCl}_{2}(0.12-0.14), \mathrm{HCCH}(6-31 \mathrm{G}(\mathrm{d}) 0.14), \mathrm{CH} 2=\mathrm{C}=\mathrm{CHCl}(0.10-0.11) \\
\mathrm{SOCl}_{2}(6-31 \mathrm{G}(\mathrm{d}), 6-31+\mathrm{G}(\mathrm{d}, \mathrm{p}) \text { and } 6-311+\mathrm{G}(\mathrm{d}, \mathrm{p}) 0.11)\end{array}$ \\
\hline B1-B95 & $\begin{array}{l}\mathrm{CH} 2=\mathrm{C}=\mathrm{CHCl}(0.11-0.12), \mathrm{HCCCCH}(6-31 \mathrm{G}(\mathrm{d}, \mathrm{p}) \text { and } 6-31+\mathrm{G}(\mathrm{d}, \mathrm{p}) 0.10-0.12), \mathrm{CSCl}_{2}(0.10- \\
0.12), \mathrm{HCCF}(631 \mathrm{G}(2 \mathrm{df}, \mathrm{p}) 0.10)\end{array}$ \\
\hline B1-LYP & $\begin{array}{l}\mathrm{HCCCCH}(6-31 \mathrm{G}(\mathrm{d}) \text { and } 6-31+\mathrm{G}(\mathrm{d}, \mathrm{p}) 0.11-0.13), \mathrm{CSCl}_{2}(0.11-0.12), \mathrm{ClCCCl}(6-311+\mathrm{G}(\mathrm{d}, \mathrm{p}) \text { and } \\
6-311+\mathrm{G}(2 \mathrm{df}, \mathrm{p}) 0.10-0.12), \mathrm{CH} 2=\mathrm{C}=\mathrm{CHCl}(0.11), \mathrm{HCCF}(6-311+\mathrm{G}(\mathrm{d}, \mathrm{p}) 0.10)\end{array}$ \\
\hline B3-LYP & $\begin{array}{l}\mathrm{ClCCCl}(6-311+\mathrm{G}(\mathrm{d}, \mathrm{p}) 0.14), \mathrm{CSCl}_{2}(0.11-0.13), \mathrm{HCCCCH}(6-31 \mathrm{G}(\mathrm{d}) \text { and } 6-31+\mathrm{G}(\mathrm{d}, \mathrm{p}) 0.10- \\
0.13), \mathrm{CH} 2=\mathrm{C}=\mathrm{CHCl}(0.10-0.11), \mathrm{ClF}_{3}(6-311+\mathrm{G}(\mathrm{d}, \mathrm{p}) 0.11)\end{array}$ \\
\hline B3-P86 & $\begin{array}{l}\mathrm{ClCCCl}(6-31+\mathrm{G}(\mathrm{d}, \mathrm{p}) \text { and } 6-311+\mathrm{G}(\mathrm{d}, \mathrm{p}) 0.10-0.16), \mathrm{CSCl}_{2}(0.11-0.13), \mathrm{CH} 2=\mathrm{C}=\mathrm{CHCl}(0.11- \\
0.12), \mathrm{HCCCCH}(6-31 \mathrm{G}(\mathrm{d}) \text { and } 6-31+\mathrm{G}(\mathrm{d}, \mathrm{p}) 0.11-0.12)\end{array}$ \\
\hline B3-PW91 & $\begin{array}{l}\mathrm{ClCCCl}(6-31+\mathrm{G}(\mathrm{d}, \mathrm{p}) \text { and } 6-311+\mathrm{G}(\mathrm{d}, \mathrm{p}) 0.10-0.15), \mathrm{CSCl}_{2}(0.11-0.13), \mathrm{CH} 2=\mathrm{C}=\mathrm{CHCl}(0.11- \\
0.12), \mathrm{HCCCCH}(6-31 \mathrm{G}(\mathrm{d}) \text { and } 6-31+\mathrm{G}(\mathrm{d}, \mathrm{p}) 0.10-0.12)\end{array}$ \\
\hline B971 & $\begin{array}{l}\mathrm{ClCCCl}(6-31 \mathrm{G}(\mathrm{d}), 6-31+\mathrm{G}(\mathrm{d}, \mathrm{p}) \text { and } 6-311+\mathrm{G}(\mathrm{d}, \mathrm{p}) 0.11-0.19), \mathrm{HCCCCH}(6-31 \mathrm{G}(\mathrm{d}) \text { and 6- } \\
31+\mathrm{G}(\mathrm{d}, \mathrm{p}) 0.10-0.13), \mathrm{CSCl}_{2}(0.11-0.12), \mathrm{CH} 2=\mathrm{C}=\mathrm{CHCl}(0.11-0.12)\end{array}$ \\
\hline B972 & $\begin{array}{l}\mathrm{ClCCCl}(6-31+\mathrm{G}(\mathrm{d}, \mathrm{p}) \text { and } 6-311+\mathrm{G}(\mathrm{d}, \mathrm{p}) 0.10-0.15), \mathrm{CSCl}_{2}(0.11-0.13), \mathrm{CH} 2=\mathrm{C}=\mathrm{CHCl}(0.11- \\
0.12), \mathrm{HCCCCH}(6-31+\mathrm{G}(\mathrm{d}, \mathrm{p}) 0.11)\end{array}$ \\
\hline B98 & $\begin{array}{l}\mathrm{ClCCCl}(6-31 \mathrm{G}(\mathrm{d}), 6-31+\mathrm{G}(\mathrm{d}, \mathrm{p}) \text { and } 6-311+\mathrm{G}(\mathrm{d}, \mathrm{p}) 0.11-0.17), \mathrm{CSCl}_{2}(0.10-0.13), \mathrm{HCCCCH}(6- \\
31 \mathrm{G}(\mathrm{d}) \text { and } 6-31+\mathrm{G}(\mathrm{d}, \mathrm{p}) 0.11-0.13), \mathrm{CH} 2=\mathrm{C}=\mathrm{CHCl}(0.11-0.12)\end{array}$ \\
\hline BB1K & $\mathrm{CH} 2=\mathrm{C}=\mathrm{CHCl}(0.12), \mathrm{HCCCCH}(0.10-0.12), \mathrm{CSCl}_{2}(0.11)$ \\
\hline BHandH & $\begin{array}{l}\mathrm{ClCCCl}(6-31 \mathrm{G}(2 \mathrm{df}, \mathrm{p}) \text { and } 6-311+\mathrm{G}(2 \mathrm{df}, \mathrm{p}) 0.10-0.14), \mathrm{HCCCCH}(6-31 \mathrm{G}(\mathrm{d}) \text { and } 6-31+\mathrm{G}(\mathrm{d}, \mathrm{p}) \\
0.11-0.13), \mathrm{HCCF}(631 \mathrm{G}(\mathrm{d}), 6-31+\mathrm{G}(\mathrm{d}, \mathrm{p}) 6-311+\mathrm{G}(\mathrm{d}, \mathrm{p}) \text { and } 6-311+\mathrm{G}(2 \mathrm{df}, \mathrm{p}) 0.10-0.13) \\
\mathrm{CH} 2=\mathrm{C}=\mathrm{CHCl}(0.11-0.12), \mathrm{CSCl}_{2}(0.10-0.12), \mathrm{ONF}(6-31 \mathrm{G}(\mathrm{d}) \text { and } 6-31 \mathrm{G}(2 \mathrm{df}, \mathrm{p}) 0.12)\end{array}$ \\
\hline BHandH- & HCCCCH (6-31G(d) and 6-31+G(d,p) 0.11-0.13), HCCF (6-31+G(d,p), 6-31G(2df,p), 6- \\
\hline & $311+\mathrm{G}(\mathrm{d}, \mathrm{p})$ and $6-311+\mathrm{G}(2 \mathrm{df}, \mathrm{p}) 0.10-0.13), \mathrm{CH} 2=\mathrm{C}=\mathrm{CHCl}(0.11-12), \mathrm{CSCl}_{2}(0.10-0.11)$ \\
\hline BMK & $\begin{array}{l}\mathrm{HCCCCH}(6-31+\mathrm{G}(\mathrm{d}, \mathrm{p}) \text { and } 6-31 \mathrm{G}(\mathrm{d}) 0.14-0.16), \mathrm{ClCCCl}(6-31 \mathrm{G}(\mathrm{d}), 6-31+\mathrm{G}(\mathrm{d}, \mathrm{p}) \text { and 6- } \\
311+\mathrm{G}(\mathrm{d}, \mathrm{p}) 0.11-0.15), \mathrm{CH} 2=\mathrm{C}=\mathrm{CHCl}(0.11-0.12), \mathrm{CSCl}_{2}(6-31 \mathrm{G}(\mathrm{d}), 6-31+\mathrm{G}(\mathrm{d}, \mathrm{p}) \text { and 6- } \\
311+\mathrm{G}(\mathrm{d}, \mathrm{p}) 0.10)\end{array}$ \\
\hline EDF1 & $\begin{array}{l}\mathrm{ClCCCl}(0.21-0.30), \mathrm{ClF}_{3}(0.11-0.20), \mathrm{CSCl}_{2}(6-31 \mathrm{G}(\mathrm{d}) \text { and } 6-31+\mathrm{G}(\mathrm{d}, \mathrm{p}) 0.13), \mathrm{F}_{2} \mathrm{SO}(0.09-0.12), \\
\mathrm{HCCH}(6-31 \mathrm{G}(\mathrm{d}) 0.13), \mathrm{HCCCCH}(6-31+\mathrm{G}(\mathrm{d}, \mathrm{p}) 0.12), \mathrm{CH} 2=\mathrm{C}=\mathrm{CHCl}(0.11)\end{array}$ \\
\hline EDF2 & $\begin{array}{l}\mathrm{BH}_{3} \mathrm{CO}(6-311+\mathrm{G}(\mathrm{d}, \mathrm{p}) 0.19), \mathrm{ClCCCl}(6-31+\mathrm{G}(\mathrm{d}, \mathrm{p}) \text { and } 6-311+\mathrm{G}(\mathrm{d}, \mathrm{p}) 0.10-0.15), \mathrm{CSCl}_{2}(0.12- \\
0.13), \mathrm{HCCCCH}(6-31 \mathrm{G}(\mathrm{d}) \text { and } 6-31+\mathrm{G}(\mathrm{d}, \mathrm{p}) 0.10-0.13), \mathrm{ClF}_{3}(6-311+\mathrm{G}(\mathrm{d}, \mathrm{p}) 0.11), \mathrm{CH} 2=\mathrm{C}=\mathrm{CHCl} \\
(0.11)\end{array}$ \\
\hline M05 & $\begin{array}{l}\mathrm{ClCCCl}(6-31 \mathrm{G}(\mathrm{d}), 6-31+\mathrm{G}(\mathrm{d}, \mathrm{p}) \text { and } 6-311+\mathrm{G}(\mathrm{d}, \mathrm{p}) 0.11-0.14), \mathrm{CH} 2=\mathrm{C}=\mathrm{CHCl}(0.12-0.13), \mathrm{CSCl}_{2} \\
(0.10-0.13), \mathrm{HCCCCH}(6-31 \mathrm{G}(\mathrm{d}) \text { and } 6-31+\mathrm{G}(\mathrm{d}, \mathrm{p}) 0.10-0.12), \mathrm{HCCF}(6-31 \mathrm{G}(2 \mathrm{df}, \mathrm{p}) \text { and } 6- \\
311+\mathrm{G}(\mathrm{d}, \mathrm{p}) 0.10-0.11)\end{array}$ \\
\hline M05-2x & $\begin{array}{l}\mathrm{HCCCCH}\left(6-31 \mathrm{G}(\mathrm{d}) \text { and 6-31+G(d,p) 0.12-0.13), } \mathrm{CH} 2=\mathrm{C}=\mathrm{CHCl}(0.11-0.12), \mathrm{C}_{2} \mathrm{~N}_{2}(6-\right. \\
311+\mathrm{G}(\mathrm{d}, \mathrm{p}) \text { and } 6-311+\mathrm{G}(2 \mathrm{df}, \mathrm{p}) 0.10-0.12), \mathrm{ClCCCl}(6-311+\mathrm{G}(2 \mathrm{df}, \mathrm{p}) 0.12), \mathrm{CSCl}_{2}(0.10-0.11)\end{array}$ \\
\hline MPW1K & $\begin{array}{l}\mathrm{HCCF}(6-31+\mathrm{G}(\mathrm{d}, \mathrm{p}), 6-31 \mathrm{G}(2 \mathrm{df}, \mathrm{p}), 6-311+\mathrm{G}(\mathrm{d}, \mathrm{p}) \text { and } 6-311+\mathrm{G}(2 \mathrm{df}, \mathrm{p}) 0.10-0.13), \mathrm{CH} 2=\mathrm{C}=\mathrm{CHCl} \\
(0.12), \mathrm{CSCl}_{2}(0.10-0.12), \mathrm{HCCCH}(6-31 \mathrm{G}(\mathrm{d}) \text { and } 6-31+\mathrm{G}(\mathrm{d}, \mathrm{p}) 0.11-0.12), \mathrm{ClCCCl}(6- \\
311+\mathrm{G}(2 \mathrm{df}, \mathrm{p}) 0.12)\end{array}$ \\
\hline MPW1PW91 & $\begin{array}{l}\mathrm{CSCl}_{2}(0.11-0.13), \mathrm{ClCCCl}(6-311+\mathrm{G}(\mathrm{d}, \mathrm{p}) \text { and 6-311+G(2df,p) } 0.10-0.13), \mathrm{CH} 2=\mathrm{C}=\mathrm{CHCl}(0.11- \\
0.12), \mathrm{HCCCCH}(6-31 \mathrm{G}(\mathrm{d}) \text { and } 6-31+\mathrm{G}(\mathrm{d}, \mathrm{p}) 0.10-0.12), \mathrm{HCCF}(6-31 \mathrm{G}(2 \mathrm{df}, \mathrm{p}) \text { and } 6-311+\mathrm{G}(\mathrm{d}, \mathrm{p}) \\
0.10)\end{array}$ \\
\hline MPWB1K & $\begin{array}{l}\mathrm{HCCF}(6-31 \mathrm{G}(2 \mathrm{df}, \mathrm{p}) 0.13), \mathrm{CH} 2=\mathrm{C}=\mathrm{CHCl}(0.12), \mathrm{HCCCCH}(6-31 \mathrm{G}(\mathrm{d}) \text { and } 6-31+\mathrm{G}(\mathrm{d}, \mathrm{p}) 0.10- \\
0.12), \mathrm{CSCl}_{2}(0.10-0.11)\end{array}$ \\
\hline O3-LYP & $\begin{array}{l}\mathrm{ClCCCl}(6-31 \mathrm{G}(\mathrm{d}), 6-31+\mathrm{G}(\mathrm{d}, \mathrm{p}) \text { and } 6-311+\mathrm{G}(\mathrm{d}, \mathrm{p}) 0.13-0.21), \mathrm{CSCl}_{2}(0.11-0.13), \mathrm{CH} 2=\mathrm{C}=\mathrm{CHCl} \\
(0.11-0.12), \mathrm{ClF}_{3}(6-311+\mathrm{G}(\mathrm{d}, \mathrm{p}) 0.12), \mathrm{HCCCCH}(6-31+\mathrm{G}(\mathrm{d}, \mathrm{p}) 0.11)\end{array}$ \\
\hline PBE1PBE & $\begin{array}{l}\mathrm{CSCl}_{2}(0.11-0.13), \mathrm{ClCCCl}(6-311+\mathrm{G}(\mathrm{d}, \mathrm{p}) \text { and } 6-311+\mathrm{G}(2 \mathrm{df}, \mathrm{p}) 0.10-0.13), \mathrm{CH} 2=\mathrm{C}=\mathrm{CHCl}(0.11- \\
0.12), \mathrm{HCCCCH}(6-31+\mathrm{G}(\mathrm{d}, \mathrm{p}) \text { and } 6-31 \mathrm{G}(\mathrm{d}) 0.10-0.12), \mathrm{HCCF}(6-31 \mathrm{G}(2 \mathrm{df}, \mathrm{p}) \text { and } 6-311+\mathrm{G}(\mathrm{d}, \mathrm{p}) \\
0.10)\end{array}$ \\
\hline
\end{tabular}




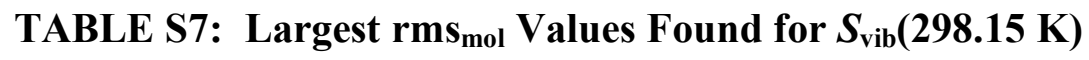

\begin{tabular}{|c|c|}
\hline Method & Largest $\mathrm{rms}_{\mathrm{mol}}$ values $\left(\mathrm{J} \mathrm{K}^{-1} \mathrm{~mol}^{-1}\right)$ \\
\hline HF & $\begin{array}{l}\mathrm{ClCCCl}(6-31 \mathrm{G}(2 \mathrm{df}, \mathrm{p}) \text { and 6-311+G(2df,p) } 0.93-1.07), \mathrm{HCCF}(6-31+\mathrm{G}(\mathrm{d}, \mathrm{p}), 6-31 \mathrm{G}(2 \mathrm{df}, \mathrm{p}), 6- \\
311+\mathrm{G}(\mathrm{d}, \mathrm{p}) \text { and } 6-311+\mathrm{G}(2 \mathrm{df}, \mathrm{p}) 0.81-0.97), \mathrm{CSCl}_{2}(6-31 \mathrm{G}(\mathrm{d}), 6-31+\mathrm{G}(\mathrm{d}, \mathrm{p}) \text { and } 6-311+\mathrm{G}(\mathrm{d}, \mathrm{p}) \\
0.85-0.86)\end{array}$ \\
\hline MP2 & $\begin{array}{l}\mathrm{CH}_{3} \mathrm{CCCH}_{3}(6-31 \mathrm{G}(\mathrm{d}) \text { and } 6-31+\mathrm{G}(\mathrm{d}, \mathrm{p}) 1.43), \mathrm{HCCH}(6-31 \mathrm{G}(\mathrm{d}) 1.36), \mathrm{CSCl}_{2}(0.89-1.16), \mathrm{CINS} \\
(6-31 \mathrm{G}(\mathrm{d}), 6-31+\mathrm{G}(\mathrm{d}, \mathrm{p}) \text { and } 6-311+\mathrm{G}(\mathrm{d}, \mathrm{p}) 0.96-0.107), \mathrm{CH}_{3} \mathrm{CCH}(6-31 \mathrm{G}(\mathrm{d}) 0.93), \mathrm{NSF}(6- \\
311+\mathrm{G}(\mathrm{d}, \mathrm{p}) 0.90)\end{array}$ \\
\hline QCISD & $\begin{array}{l}\mathrm{CSCl}_{2}(1.04-1.07), \mathrm{HCCH}(6-31 \mathrm{G}(\mathrm{d}) 1.06), \mathrm{CH}_{3} \mathrm{CCCH}_{3}(6-31 \mathrm{G}(\mathrm{d}) \text { and } 6-31+\mathrm{G}(\mathrm{d}, \mathrm{p}) 0.89-0.93) \\
\mathrm{CH} 2=\mathrm{C}=\mathrm{CHCl}(6-31+\mathrm{G}(\mathrm{d}, \mathrm{p}) \text { and } 6-311+\mathrm{G}(\mathrm{d}, \mathrm{p}) 0.81-0.83)\end{array}$ \\
\hline QCISD(T) & $\begin{array}{l}\mathrm{HCCH}(6-31 \mathrm{G}(\mathrm{d}) \text { and } 6-31+\mathrm{G}(\mathrm{d}, \mathrm{p}) 1.05-1.70), \mathrm{CH}_{3} \mathrm{CCCH}_{3}(6-31 \mathrm{G}(\mathrm{d}) \text { and } 6-31+\mathrm{G}(\mathrm{d}, \mathrm{p}) 1.35-1.43) \text {, } \\
\mathrm{CSCl}_{2}(1.12-1.16), \mathrm{CH}_{3} \mathrm{CCH}(0.88-1.01), \mathrm{HCCCH}_{2} \mathrm{Cl}(6-31+\mathrm{G}(\mathrm{d}, \mathrm{p}) 0.96), \mathrm{CH} 2=\mathrm{C}=\mathrm{CHCl}(6- \\
31+\mathrm{G}(\mathrm{d}, \mathrm{p}) \text { and } 6-311+\mathrm{G}(\mathrm{d}, \mathrm{p}) 0.86-0.88)\end{array}$ \\
\hline CCSD & $\begin{array}{l}\mathrm{CSCl}_{2}(1.03-1.06), \mathrm{HCCH}(6-31 \mathrm{G}(\mathrm{d}) 1.03), \mathrm{CH}_{3} \mathrm{CCCH}_{3}(6-31 \mathrm{G}(\mathrm{d}) \text { and } 6-31+\mathrm{G}(\mathrm{d}, \mathrm{p}) 0.89-0.93) \\
\mathrm{CH} 2=\mathrm{C}=\mathrm{CHCl}(6-31+\mathrm{G}(\mathrm{d}, \mathrm{p}) \text { and } 6-311+\mathrm{G}(\mathrm{d}, \mathrm{p}) 0.81-0.83)\end{array}$ \\
\hline $\operatorname{CCSD}(\mathrm{T})$ & $\begin{array}{l}\mathrm{HCCH}(6-31 \mathrm{G}(\mathrm{d}) \text { and } 6-31+\mathrm{G}(\mathrm{d}, \mathrm{p}) 1.03-1.67), \mathrm{CH}_{3} \mathrm{CCCH}_{3}(6-31 \mathrm{G}(\mathrm{d}) \text { and } 6-31+\mathrm{G}(\mathrm{d}, \mathrm{p}) 1.35-1.43) \text {, } \\
\mathrm{CSCl}_{2}(1.11-1.15), \mathrm{CH}_{3} \mathrm{CCH}(0.86-0.99), \mathrm{HCCCH}_{2} \mathrm{Cl}(6-31+\mathrm{G}(\mathrm{d}, \mathrm{p}) 0.95), \mathrm{CH}=\mathrm{C}=\mathrm{CHCl}(6- \\
31+\mathrm{G}(\mathrm{d}, \mathrm{p}) \text { and } 6-311+\mathrm{G}(\mathrm{d}, \mathrm{p}) 0.86-0.88)\end{array}$ \\
\hline B-B95 & $\mathrm{ClCCCl}(6-31 \mathrm{G}(\mathrm{d})$ and 6-31+G(d,p) $1.80-2.09), \mathrm{CSCl}_{2}(0.90-1.04), \mathrm{ClF}_{3}(6-31+\mathrm{G}(\mathrm{d}, \mathrm{p}) 1.02)$ \\
\hline B-LYP & $\begin{array}{l}\mathrm{ClCCCl}(6-31 \mathrm{G}(\mathrm{d}), 6-31+\mathrm{G}(\mathrm{d}, \mathrm{p}) \text { and } 6-311+\mathrm{G}(\mathrm{d}, \mathrm{p}) 1.76-2.54), \mathrm{ClF}_{3}(6-31 \mathrm{G}(\mathrm{d}), 6-31+\mathrm{G}(\mathrm{d}, \mathrm{p}), 6- \\
311+\mathrm{G}(\mathrm{d}, \mathrm{p}) \text { and } 6-311+\mathrm{G}(2 \mathrm{df}, \mathrm{p}) 0.92-1.57), \mathrm{CSCl}_{2}(0.93-1.08), \mathrm{SOCl}_{2}(6-31 \mathrm{G}(\mathrm{d}), 6-31+\mathrm{G}(\mathrm{d}, \mathrm{p}) \\
\text { and } 6-311+\mathrm{G}(\mathrm{d}, \mathrm{p}) 0.80-0.86)\end{array}$ \\
\hline B-P86 & $\begin{array}{l}\mathrm{ClCCCl}(6-31 \mathrm{G}(\mathrm{d}), 6-31+\mathrm{G}(\mathrm{d}, \mathrm{p}) \text { and } 6-311+\mathrm{G}(\mathrm{d}, \mathrm{p}) 1.89-2.68), \mathrm{ClF}_{3}(6-31+\mathrm{G}(\mathrm{d}, \mathrm{p}) \text { and } 6- \\
311+\mathrm{G}(\mathrm{d}, \mathrm{p}) 0.95-1.21), \mathrm{CSCl}_{2}(0.92-1.10)\end{array}$ \\
\hline G96-LYP & $\begin{array}{l}\mathrm{ClCCCl}\left(6-31 \mathrm{G}(\mathrm{d}), 6-31+\mathrm{G}(\mathrm{d}, \mathrm{p}) \text { and 6-311+G(d,p) 1.77-2.43), } \mathrm{ClF}_{3}(6-31 \mathrm{G}(\mathrm{d}), 6-31+\mathrm{G}(\mathrm{d}, \mathrm{p}), 6-\right. \\
311+\mathrm{G}(\mathrm{d}, \mathrm{p}) \text { and 6-311+G(2df,p) } 0.87-1.50), \mathrm{CSCl}_{2}(0.92-1.07), \mathrm{HCCCCH}(6-31+\mathrm{G}(\mathrm{d}, \mathrm{p}) 0.83)\end{array}$ \\
\hline НCTH147 & $\begin{array}{l}\mathrm{ClCCCl}(6-31 \mathrm{G}(\mathrm{d}), 6-31+\mathrm{G}(\mathrm{d}, \mathrm{p}) \text { and } 6-311+\mathrm{G}(\mathrm{d}, \mathrm{p}) 1.66-2.44), \mathrm{CSCl}_{2}(0.94-1.10), \mathrm{ClF}_{3}(6- \\
311+\mathrm{G}(\mathrm{d}, \mathrm{p}) \text { and } 6-31+\mathrm{G}(\mathrm{d}, \mathrm{p}) 0.82-1.08)\end{array}$ \\
\hline НCTH407 & $\begin{array}{l}\mathrm{ClCCCl}(6-31 \mathrm{G}(\mathrm{d}), 6-31+\mathrm{G}(\mathrm{d}, \mathrm{p}) \text { and } 6-311+\mathrm{G}(\mathrm{d}, \mathrm{p}) 1.40-2.26), \mathrm{CSCl}_{2}(0.96-1.12), \mathrm{ClF}_{3}(6- \\
311+\mathrm{G}(\mathrm{d}, \mathrm{p}) 1.06)\end{array}$ \\
\hline НСТН93 & $\begin{array}{l}\mathrm{ClCCCl}(6-31 \mathrm{G}(\mathrm{d}), 6-31+\mathrm{G}(\mathrm{d}, \mathrm{p}) \text { and } 6-311+\mathrm{G}(\mathrm{d}, \mathrm{p}) 1.24-1.98), \mathrm{ClF}_{3}(6-311+\mathrm{G}(\mathrm{d}, \mathrm{p}) \text { and 6- } \\
31+\mathrm{G}(\mathrm{d}, \mathrm{p}) 0.91-1.21), \mathrm{CSCl}_{2}(0.90-1.03)\end{array}$ \\
\hline O-LYP & $\begin{array}{l}\mathrm{ClCCCl}(6-31 \mathrm{G}(\mathrm{d}), 6-31+\mathrm{G}(\mathrm{d}, \mathrm{p}) \text { and } 6-311+\mathrm{G}(\mathrm{d}, \mathrm{p}) 1.83-2.46), \mathrm{ClF}_{3}(6-31+\mathrm{G}(\mathrm{d}, \mathrm{p}) \text { and 6- } \\
311+\mathrm{G}(\mathrm{d}, \mathrm{p}) 0.85-1.13), \mathrm{CSCl}_{2}(0.94-1.11)\end{array}$ \\
\hline PBEPBE & $\begin{array}{l}\mathrm{ClCCCl}(6-31 \mathrm{G}(\mathrm{d}), 6-31+\mathrm{G}(\mathrm{d}, \mathrm{p}) \text { and } 6-311+\mathrm{G}(\mathrm{d}, \mathrm{p}) 1.72-2.58), \mathrm{ClF}_{3}(6-31+\mathrm{G}(\mathrm{d}, \mathrm{p}) \text { and } 6- \\
311+\mathrm{G}(\mathrm{d}, \mathrm{p}) 0.90-1.16), \mathrm{CSCl}_{2}(0.92-1.10)\end{array}$ \\
\hline TPSSTPSS & $\begin{array}{l}\mathrm{ClCCCl}(6-31 \mathrm{G}(\mathrm{d}), 6-31+\mathrm{G}(\mathrm{d}, \mathrm{p}) \text { and } 6-311+\mathrm{G}(\mathrm{d}, \mathrm{p}) 1.71-2.41), \mathrm{CSCl}_{2}(0.89-1.06), \mathrm{ClF}_{3}(6- \\
31+\mathrm{G}(\mathrm{d}, \mathrm{p}) \text { and } 6-311+\mathrm{G}(\mathrm{d}, \mathrm{p}) 0.81-1.01)\end{array}$ \\
\hline VSXC & $\begin{array}{l}\mathrm{ClCCCl}\left(6-31 \mathrm{G}(\mathrm{d}), 6-31+\mathrm{G}(\mathrm{d}, \mathrm{p}) \text { and 6-311+G(d,p) 1.37-2.19), } \mathrm{CSCl}_{2}(0.97-1.08), \mathrm{HCOOCH}_{3}(6-\right. \\
31 \mathrm{G}(2 \mathrm{df}, \mathrm{p}), 6-311+\mathrm{G}(\mathrm{d}, \mathrm{p}) \text { and } 6-311+\mathrm{G}(2 \mathrm{df}, \mathrm{p}) 0.96-1.04), \mathrm{ClF}_{3}(6-311+\mathrm{G}(\mathrm{d}, \mathrm{p}) 0.92)\end{array}$ \\
\hline B1-B95 & $\mathrm{CSCl}_{2}(0.83-0.95)$ \\
\hline B1-LYP & $\mathrm{CSCl}_{2}(0.85-0.98), \mathrm{ClCCCl}(6-311+\mathrm{G}(\mathrm{d}, \mathrm{p}) \quad 0.82)$ \\
\hline B3-LYP & $\mathrm{ClCCCl}(6-311+\mathrm{G}(\mathrm{d}, \mathrm{p}) 1.01), \mathrm{CSCl}_{2}(0.86-1.00)$ \\
\hline B3-P86 & $\mathrm{ClCCCl}(6-311+\mathrm{G}(\mathrm{d}, \mathrm{p}) 1.13), \mathrm{CSCl}_{2}(0.86-1.01)$ \\
\hline B3-PW91 & $\mathrm{ClCCCl}(6-311+\mathrm{G}(\mathrm{d}, \mathrm{p}) 1.06), \mathrm{CSCl}_{2}(0.86-1.01)$ \\
\hline B971 & $\mathrm{ClCCCl}(6-31 \mathrm{G}(\mathrm{d}), 6-31+\mathrm{G}(\mathrm{d}, \mathrm{p})$ and $6-311+\mathrm{G}(\mathrm{d}, \mathrm{p}) 0.81-1.38), \mathrm{CSCl}_{2}(0.85-0.98)$ \\
\hline B972 & $\mathrm{ClCCCl}(6-311+\mathrm{G}(\mathrm{d}, \mathrm{p}) 1.06), \mathrm{CSCl}_{2}(0.88-1.02)$ \\
\hline B98 & $\mathrm{ClCCCl}(6-31+\mathrm{G}(\mathrm{d}, \mathrm{p})$ and $6-311+\mathrm{G}(\mathrm{d}, \mathrm{p}) 0.87-1.26), \mathrm{CSCl}_{2}(0.84-0.97)$ \\
\hline BB1K & $\mathrm{CSCl}_{2}(0.90-0.91)$ \\
\hline BHandH & $\mathrm{CSCl}_{2}(0.81-0.94) \mathrm{ClCCCl}(6-311+\mathrm{G}(2 \mathrm{df}, \mathrm{p}) 0.91)$ \\
\hline
\end{tabular}




\begin{tabular}{|c|c|}
\hline $\begin{array}{l}\text { BHandH- } \\
\text { LYP }\end{array}$ & $\mathrm{CSCl}_{2}(0.81-0.92) \mathrm{ClCCCl}(6-311+\mathrm{G}(2 \mathrm{df}, \mathrm{p}) 0.87)$ \\
\hline $\mathrm{BMK}$ & $\begin{array}{l}\mathrm{CSCl}_{2}(6-31 \mathrm{G}(\mathrm{d}), 6-31+\mathrm{G}(\mathrm{d}, \mathrm{p}) \text { and } 6-311+\mathrm{G}(\mathrm{d}, \mathrm{p}) \text { 0.86-0.89), } \mathrm{HCCCCH}(6-31+\mathrm{G}(\mathrm{d}) \text { and 6-31G(d) } \\
0.83-0.88)\end{array}$ \\
\hline EDF1 & $\begin{array}{l}\mathrm{ClCCCl}(1.61-2.42), \mathrm{ClF}_{3}\left(6-31+\mathrm{G}(\mathrm{d}, \mathrm{p}), \text { and 6-311+G(d,p) 1.00-1.30), } \mathrm{CSCl}_{2}(6-31 \mathrm{G}(\mathrm{d}) \text { and } 6-\right. \\
31+\mathrm{G}(\mathrm{d}, \mathrm{p}) 1.03-1.05)\end{array}$ \\
\hline EDF2 & $\mathrm{BH}_{3} \mathrm{CO}(6-311+\mathrm{G}(\mathrm{d}, \mathrm{p}) 1.42), \mathrm{ClCCCl}(6-311+\mathrm{G}(\mathrm{d}, \mathrm{p}) 1.05), \mathrm{CSCl}_{2}(0.94-1.01)$ \\
\hline M05 & $\mathrm{CSCl}_{2}(0.81-0.98), \mathrm{ClCCCl}(6-31 \mathrm{G}(\mathrm{d}), 6-31+\mathrm{G}(\mathrm{d}, \mathrm{p})$ and $6-311+\mathrm{G}(\mathrm{d}, \mathrm{p}) 0.83-0.98)$ \\
\hline M05-2x & $\begin{array}{l}\mathrm{CH}_{3} \mathrm{COF}(6-31 \mathrm{G}(2 \mathrm{df}, \mathrm{p}), 6-311+\mathrm{G}(\mathrm{d}, \mathrm{p}) \text { and 6-311+G(2df,p) } 1.00-1.10), \mathrm{CH}_{3} \mathrm{CHO}(6-31+\mathrm{G}(\mathrm{d}, \mathrm{p}), 6- \\
31 \mathrm{G}(2 \mathrm{df}, \mathrm{p}), 6-311+\mathrm{G}(\mathrm{d}, \mathrm{p}) \text { and } 6-311+\mathrm{G}(2 \mathrm{df}, \mathrm{p}) 0.81-1.00), \mathrm{O}=\mathrm{CHCH}=\mathrm{O}(6-31+\mathrm{G}(\mathrm{d}, \mathrm{p}) \text { and 6- } \\
311+\mathrm{G}(2 \mathrm{df}, \mathrm{p}) 0.94-0.96), \mathrm{HCOOCH}_{3}(0.79-0.89), \mathrm{CSCl}_{2}(6-31 \mathrm{G}(\mathrm{d}), 6-31+\mathrm{G}(\mathrm{d}, \mathrm{p}) \text { and 6- } \\
311+\mathrm{G}(\mathrm{d}, \mathrm{p}) 0.87-0.88)\end{array}$ \\
\hline MPW1K & $\mathrm{CSCl}_{2}(0.82-0.95), \mathrm{ClCCCl}(6-311+\mathrm{G}(2 \mathrm{df}, \mathrm{p}) \quad 0.84)$ \\
\hline MPW1PW91 & $\mathrm{CSCl}_{2}(0.85-1.00), \mathrm{ClCCCl}(6-311+\mathrm{G}(\mathrm{d}, \mathrm{p}) 0.88)$ \\
\hline MPWB1K & $\mathrm{CSCl}_{2}(0.80-0.91)$ \\
\hline O3-LYP & $\mathrm{ClCCCl}\left(6-31 \mathrm{G}(\mathrm{d}), 6-31+\mathrm{G}(\mathrm{d}, \mathrm{p})\right.$ and $6-311+\mathrm{G}(2 \mathrm{df}, \mathrm{p})$ 0.96-1.56), $\mathrm{CSCl}_{2}(0.90-1.04)$ \\
\hline PBE1PBE & $\mathrm{CSCl}_{2}(0.85-1.00), \mathrm{ClCCCl}(6-311+\mathrm{G}(\mathrm{d}, \mathrm{p}) 0.95)$ \\
\hline
\end{tabular}




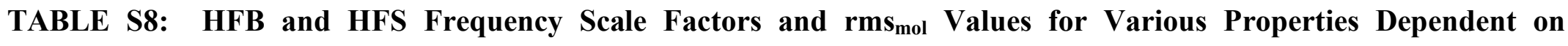
Frequency $^{a}$

\begin{tabular}{|c|c|c|c|c|c|c|c|c|c|c|}
\hline \multirow[b]{2}{*}{ Method } & \multicolumn{2}{|l|}{$\omega^{b}$} & \multicolumn{2}{|l|}{$1 / \omega^{c}$} & \multicolumn{2}{|c|}{$\mathrm{ZPVE}^{d}$} & \multicolumn{2}{|c|}{$\Delta H_{\mathrm{vib}}(298.15 \mathrm{~K})^{c}$} & \multicolumn{2}{|c|}{$S_{\mathrm{vib}}(298.15 \mathrm{~K})^{c}$} \\
\hline & scale factor ${ }^{e}$ & $\mathrm{rms}_{\mathrm{ov}}{ }^{f}$ & scale factor ${ }^{g}$ & $\mathrm{rms}_{\mathrm{ov}}{ }^{h}$ & scale factor ${ }^{i}$ & $\mathrm{rms}_{\mathrm{ov}}^{j}$ & scale factor ${ }^{k}$ & $\mathrm{rms}_{\mathrm{ov}}{ }^{l}$ & scale factor ${ }^{m}$ & $\mathrm{rms}_{\mathrm{ov}}{ }^{n}$ \\
\hline $\mathrm{HFB} / \mathrm{A}$ & 1.0258 & 55 & 1.1157 & 22 & 1.0429 & 0.75 & 1.1182 & 0.059 & 1.1244 & 0.41 \\
\hline $\mathrm{HFB} / \mathrm{B}$ & 1.0285 & 56 & 1.1307 & 22 & 1.0463 & 0.56 & 1.1257 & 0.057 & 1.1325 & 0.40 \\
\hline $\mathrm{HFB} / \mathrm{C}$ & 1.0276 & 48 & 1.0976 & 17 & 1.0445 & 0.56 & 1.1012 & 0.047 & 1.1033 & 0.31 \\
\hline $\mathrm{HFB} / \mathrm{D}$ & 1.0315 & 57 & 1.1590 & 26 & 1.0481 & 0.56 & 1.1322 & 0.061 & 1.1462 & 0.44 \\
\hline $\mathrm{HFB} / \mathrm{E}$ & 1.0307 & 50 & 1.1035 & 15 & 1.0476 & 0.51 & 1.1054 & 0.044 & 1.1065 & 0.28 \\
\hline $\mathrm{HFS} / \mathrm{A}$ & 1.0252 & 44 & 1.0565 & 17 & $1.0486^{\circ}$ & $0.58^{\circ}$ & 1.0575 & 0.047 & 1.0597 & 0.32 \\
\hline $\mathrm{HFS} / \mathrm{B}$ & 1.0306 & 43 & 1.0705 & 16 & 1.0550 & 0.42 & 1.0646 & 0.044 & 1.0682 & 0.31 \\
\hline $\mathrm{HFS} / \mathrm{C}$ & 1.0282 & 46 & 1.0528 & 15 & 1.0522 & 0.53 & 1.0463 & 0.041 & 1.0482 & 0.27 \\
\hline HFS/D & 1.0347 & 43 & 1.0958 & 21 & 1.0582 & 0.49 & 1.0688 & 0.048 & 1.0802 & 0.35 \\
\hline $\mathrm{HFS} / \mathrm{E}$ & 1.0344 & 40 & 1.0589 & 13 & $1.0586^{p}$ & $0.48^{p}$ & 1.0478 & 0.034 & 1.0503 & 0.23 \\
\hline
\end{tabular}

${ }^{a}$ Basis sets employed: 6-31G(d) (basis set A), 6-31+G(d,p) (basis set B), 6-31G(2df,p) (basis set C), 6-311+G(d,p) (basis set D), and 6-31G(2df,p) (basis set E). ${ }^{b}$ Using F1 set of 1066 frequencies. ${ }^{c}$ Using F1' set of 1062 frequencies. ${ }^{d}$ Using the Z1 set of 89 frequencies. ${ }^{e}$ As defined by equation 2. ${ }^{f}$ Overall root-mean-square error, as defined by equation 5 , in units of $\mathrm{cm}^{-1}$. ${ }^{g}$ As defined by equation 7 . ${ }^{h}$ Overall root-mean-square error in units of $10^{-5}$ cm. ${ }^{i}$ As defined by equation $8 .{ }^{j}$ Overall $\mathrm{rms}$ error in ZPVE values in $\mathrm{kJ}$ mol ${ }^{-1}$. ${ }^{k}$ As defined by equation 13 . ${ }^{l}$ Overall root-mean-square error in $\Delta H_{\mathrm{vib}}(T)$ in units of $\mathrm{kJ} \mathrm{mol}^{-1}$. ${ }^{m}$ As defined by equation 14 . ${ }^{n}$ Overall root-mean-square error in $\mathrm{S}_{\mathrm{vib}}(T)$ in units of $\mathrm{J} \mathrm{K}^{-1}$ mol ${ }^{-1}$. ${ }^{o} \mathrm{Values}$ obtained with $\mathrm{S}_{2}$ removed from the analysis due to SCF convergence difficulties. ${ }^{p}$ Values obtained with SO removed from the analysis due to SCF convergence difficulties. 
TABLE S9: HFB and HFS Frequency Scale Factors and $\mathbf{r m s}_{\text {ov }}$ Values Derived from a Least-Squares Fit of Theoretical and Experimental $\Delta H_{\text {vib }}(T)$ and $S_{\text {vib }}(T)$ Values at $450 \mathrm{~K}$ and $600 \mathrm{~K}^{a}$

\begin{tabular}{lccccc}
\hline & \multicolumn{3}{c}{$\Delta H_{\text {vib }}(T)^{b}$} & & \multicolumn{2}{c}{$S_{\text {vib }}(T)^{b}$} \\
\cline { 1 - 2 } Method & $450 \mathrm{~K}$ & $600 \mathrm{~K}$ & & $450 \mathrm{~K}$ & $600 \mathrm{~K}$ \\
\cline { 2 - 3 } \cline { 5 - 6 } HFB/A & scale factor $^{c}$ & scale factor $^{c}$ & & scale factor $^{d}$ & scale factor $^{d}$ \\
HFB/B & 1.0908 & 1.0708 & & 1.1097 & 1.0969 \\
HFB/C & 1.1030 & 1.0854 & & 1.1196 & 1.1083 \\
HFB/D & 1.0823 & 1.0680 & & 1.0941 & 1.0854 \\
HFB/E & 1.1070 & 1.0888 & & 1.1290 & 1.1159 \\
HFS/A & 1.0904 & 1.0771 & & 1.0995 & 1.0854 \\
HFS/B & 1.0476 & 1.0413 & & 1.0542 & 1.0499 \\
HFS/C & 1.0595 & 1.0563 & & 1.0643 & 1.0617 \\
HFS/D & 1.0440 & 1.0427 & & 1.0463 & 1.0451 \\
HFS/E & 1.0628 & 1.0600 & & 1.0723 & 1.0685 \\
& 1.0499 & 1.0507 & & 1.0502 & 1.0504
\end{tabular}

\footnotetext{
${ }^{a}$ Basis sets employed: 6-31G(d) (basis set A), 6-31+G(d,p) (basis set B), 6-31G(2df,p) (basis set C), 6-311+G(d,p) (basis set D), and 6-31G(2df,p) (basis set E). ${ }^{b}$ Using F1' set of 1062 frequencies. ${ }^{c}$ As defined by equation $13 .{ }^{d} \mathrm{As}$ defined by equation 14 .
} 
TABLE S10: Effect of Basis Set on ZPVE Scale Factors and Corresponding rms $_{\text {ov }}$ Values $^{a}$

\begin{tabular}{|c|c|c|c|c|c|c|c|c|}
\hline \multirow{2}{*}{ Basis set } & \multicolumn{8}{|c|}{$\mathrm{ZPVE}^{a}$} \\
\hline & \multicolumn{2}{|c|}{ B3-LYP } & \multicolumn{2}{|c|}{ BMK } & \multicolumn{2}{|c|}{$\mathrm{MP}^{b}$} & \multicolumn{2}{|c|}{$\mathrm{HF}$} \\
\hline $6-31 G(d)$ & 0.9813 & 0.42 & 0.9709 & 0.49 & 0.9670 & 0.91 & 0.9135 & 0.71 \\
\hline $6-31+G(d)$ & 0.9829 & 0.34 & 0.9733 & 0.47 & 0.9723 & 0.93 & 0.9154 & 0.70 \\
\hline $6-31 G(d, p)$ & 0.9838 & 0.39 & 0.9750 & 0.55 & 0.9606 & 0.81 & 0.9181 & 0.73 \\
\hline $6-31+G(d, p)$ & 0.9857 & 0.30 & 0.9773 & 0.52 & 0.9657 & 0.82 & 0.9200 & 0.72 \\
\hline $6-311 G(d)$ & 0.9882 & 0.39 & 0.9767 & 0.54 & 0.9763 & 0.79 & 0.9214 & 0.71 \\
\hline $6-311+\mathrm{G}(\mathrm{d})$ & 0.9882 & 0.38 & 0.9773 & 0.52 & 0.9792 & 0.88 & 0.9222 & 0.70 \\
\hline $6-311 G(d, p)$ & 0.9888 & 0.36 & 0.9788 & 0.60 & 0.9748 & 0.52 & 0.9247 & 0.79 \\
\hline $6-311+G(d, p)$ & 0.9887 & 0.36 & 0.9785 & 0.52 & 0.9768 & 0.55 & 0.9255 & 0.77 \\
\hline $6-311+G(2 d, p)$ & 0.9898 & 0.27 & 0.9788 & 0.44 & 0.9793 & 0.63 & 0.9273 & 0.71 \\
\hline $6-311+\mathrm{G}(3 \mathrm{~d}, \mathrm{p})$ & 0.9891 & 0.31 & 0.9776 & 0.46 & 0.9783 & 0.59 & 0.9268 & 0.72 \\
\hline $6-311+G(3 d f, p)$ & 0.9883 & 0.33 & 0.9784 & 0.52 & 0.9774 & 0.45 & 0.9262 & 0.77 \\
\hline $6-311+\mathrm{G}(3 \mathrm{df}, 3 \mathrm{pd})$ & 0.9876 & 0.30 & 0.9779 & 0.50 & 0.9791 & 0.54 & 0.9265 & 0.76 \\
\hline $6-311++\mathrm{G}(3 \mathrm{df}, 3 \mathrm{pd})$ & 0.9876 & 0.31 & 0.9779 & 0.50 & 0.9793 & 0.55 & 0.9265 & 0.76 \\
\hline cc-pVDZ & 0.9931 & 0.47 & 0.9813 & 0.54 & 0.9782 & 0.54 & 0.9258 & 0.77 \\
\hline cc-pVTZ & 0.9878 & 0.35 & 0.9792 & 0.55 & 0.9771 & 0.46 & 0.9265 & 0.78 \\
\hline cc-pVQZ & 0.9882 & 0.34 & 0.9770 & 0.56 & 0.9808 & 0.48 & 0.9263 & 0.76 \\
\hline aug-cc-pVDZ & 0.9947 & 0.34 & $0.9838^{e}$ & $0.55^{e}$ & 0.9878 & 0.76 & 0.9298 & 0.73 \\
\hline aug-cc-pVTZ & 0.9884 & 0.36 & $0.9802^{e}$ & $0.52^{e}$ & 0.9822 & 0.50 & 0.9269 & 0.77 \\
\hline aug-cc-pVQZ & 0.9881 & 0.35 & $0.9777^{e}$ & $0.57^{e}$ & 0.9836 & 0.47 & 0.9264 & 0.76 \\
\hline TZV2P & 0.9873 & 0.30 & 0.9760 & 0.51 & 0.9782 & 0.77 & 0.9256 & 0.68 \\
\hline
\end{tabular}

${ }^{a}$ Using the Z1 set of 89 frequencies. ${ }^{b}$ Scale factors and $\mathrm{rms}_{\mathrm{ov}}$ values for MP2 calculated with $\mathrm{CN}$ and NO removed from the test set. ${ }^{c}$ As defined by equation $8 .{ }^{d}$ Overall rms error in ZPVEs in $\mathrm{kJ} \mathrm{mol}^{-1} .{ }^{e} \mathrm{NO}$ removed due to large $\mathrm{rms}_{\mathrm{mol}}$, which significantly improves the rms $\mathrm{ov}$ while causing a minimal change to the scale factor. 


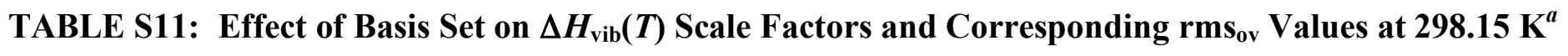

\begin{tabular}{|c|c|c|c|c|c|c|c|c|}
\hline \multirow[b]{3}{*}{ Basis set } & \multicolumn{8}{|c|}{$\Delta H_{\mathrm{vib}}(T)^{a}$} \\
\hline & \multicolumn{2}{|c|}{ B3-LYP } & \multicolumn{2}{|c|}{ BMK } & \multicolumn{2}{|c|}{$\mathrm{MP} 2^{b}$} & \multicolumn{2}{|c|}{$\mathrm{HF}$} \\
\hline & scale factor ${ }^{c}$ & $\mathrm{rms}_{\mathrm{ov}}{ }^{d}$ & scale factor ${ }^{c}$ & $\mathrm{rms}_{\mathrm{ov}}{ }^{d}$ & scale factor ${ }^{c}$ & $\mathrm{rms}_{\mathrm{ov}}{ }^{d}$ & scale factor ${ }^{c}$ & $\mathrm{rms}_{\mathrm{ov}}{ }^{d}$ \\
\hline $6-31 G(d)$ & 1.0004 & 0.035 & 0.9679 & 0.037 & 1.0059 & 0.055 & 0.8902 & 0.044 \\
\hline $6-31+G(d)$ & 1.0053 & 0.036 & 0.9721 & 0.037 & 1.0266 & 0.067 & 0.8943 & 0.044 \\
\hline $6-31 G(d, p)$ & 1.0003 & 0.035 & 0.9682 & 0.037 & 1.0003 & 0.046 & 0.8913 & 0.044 \\
\hline $6-31+G(d, p)$ & 1.0062 & 0.035 & 0.9728 & 0.037 & 1.0198 & 0.053 & 0.8955 & 0.044 \\
\hline $6-31 G(2 d f, p)$ & 0.9909 & 0.032 & 0.9610 & 0.034 & 0.9823 & 0.033 & 0.8878 & 0.044 \\
\hline $6-311 \mathrm{G}(\mathrm{d})$ & 1.0090 & 0.035 & 0.9727 & 0.034 & 1.0045 & 0.054 & 0.8947 & 0.043 \\
\hline $6-311+G(d)$ & 1.0124 & 0.036 & 0.9750 & 0.034 & 1.0206 & 0.065 & 0.8957 & 0.043 \\
\hline $6-311 \mathrm{G}(\mathrm{d}, \mathrm{p})$ & 1.0062 & 0.035 & 0.9713 & 0.034 & 0.9946 & 0.040 & 0.8951 & 0.044 \\
\hline $6-311+G(d, p)$ & 1.0102 & 0.036 & 0.9740 & 0.034 & 1.0071 & 0.044 & 0.8967 & 0.043 \\
\hline $6-311+G(2 d, p)$ & 1.0040 & 0.032 & 0.9710 & 0.030 & 1.0040 & 0.031 & 0.8964 & 0.044 \\
\hline $6-311+\mathrm{G}(2 \mathrm{df}, \mathrm{p})$ & 0.9938 & 0.031 & 0.9644 & 0.031 & 0.9876 & 0.030 & 0.8885 & 0.045 \\
\hline $6-311+\mathrm{G}(3 \mathrm{df}, \mathrm{p})$ & 0.9919 & 0.032 & 0.9623 & 0.032 & 0.9918 & 0.031 & 0.8878 & 0.045 \\
\hline $6-311+G(3 d f, 2 p)$ & 0.9915 & 0.032 & 0.9619 & 0.032 & 0.9916 & 0.030 & 0.8875 & 0.046 \\
\hline $6-311+\mathrm{G}(3 \mathrm{df}, 3 \mathrm{pd})$ & 0.9904 & 0.033 & 0.9614 & 0.033 & 0.9943 & 0.032 & 0.8864 & 0.046 \\
\hline $6-311++\mathrm{G}(3 \mathrm{df}, 3 \mathrm{pd})$ & 0.9904 & 0.033 & 0.9613 & 0.033 & 0.9947 & 0.032 & 0.8864 & 0.046 \\
\hline cc-pVDZ & 1.0061 & 0.035 & 0.9771 & 0.034 & 1.0020 & 0.035 & 0.9023 & 0.043 \\
\hline cc-pVTZ & 0.9960 & 0.030 & 0.9675 & 0.031 & 0.9919 & 0.029 & 0.8915 & 0.044 \\
\hline cc-pVQZ & 0.9946 & 0.029 & 0.9643 & 0.031 & $-e$ & $-e$ & 0.8902 & 0.043 \\
\hline aug-cc-pVDZ & $1.0266^{f}$ & $0.035^{f}$ & $0.9988^{f}$ & $0.044^{f}$ & 1.0359 & 0.048 & 0.9126 & 0.042 \\
\hline aug-cc-pVTZ & 0.9987 & 0.030 & 0.9698 & 0.031 & 1.0067 & 0.031 & 0.8929 & 0.044 \\
\hline aug-cc-pVQZ & 0.9955 & 0.029 & 0.9657 & 0.030 & $-e$ & $-e$ & 0.8908 & 0.043 \\
\hline TZV2P & 1.0058 & 0.029 & 0.9722 & 0.029 & 1.0127 & 0.037 & 0.8979 & 0.042 \\
\hline
\end{tabular}

${ }^{a}$ Using the F1' set of 1062 frequencies, unless otherwise noted. ${ }^{b}$ Using the F1" set of 1033 frequencies. ${ }^{c}$ As defined by equation $13 .{ }^{d}$ Overall rootmean-square error in units of $10^{-5} \mathrm{~cm}$. ${ }^{e} \mathrm{Scale}$ factors and $\mathrm{rms}_{\mathrm{ov}}$ values not determined at these levels of theory. ${ }^{f} \mathrm{HCCCCH}$ has a bent structure at these levels of theory, and is therefore removed from the analysis. 
TABLE S12: Effect of Basis Set on $S_{\text {vib }}(T)$ Scale Factors and Corresponding rms rv $_{\text {Values at } 298.15 \mathrm{~K}^{a}}$

\begin{tabular}{|c|c|c|c|c|c|c|c|c|}
\hline \multirow[b]{3}{*}{ basis set } & \multicolumn{8}{|c|}{$S_{\mathrm{vib}}(T)^{a}$} \\
\hline & \multicolumn{2}{|c|}{ B3-LYP } & \multicolumn{2}{|c|}{ BMK } & \multicolumn{2}{|c|}{$\mathrm{MP} 2^{b}$} & \multicolumn{2}{|c|}{$\mathrm{HF}$} \\
\hline & scale factor ${ }^{c}$ & $\mathrm{rms}_{\mathrm{ov}}{ }^{d}$ & scale factor ${ }^{c}$ & $\mathrm{rms}_{\mathrm{ov}}{ }^{d}$ & scale factor ${ }^{c}$ & $\mathrm{rms}_{\mathrm{ov}}{ }^{d}$ & scale factor ${ }^{c}$ & $\mathrm{rms}_{\mathrm{ov}}{ }^{d}$ \\
\hline $6-31 G(d)$ & 1.0029 & 0.24 & 0.9731 & 0.25 & 1.0178 & 0.39 & 0.8978 & 0.28 \\
\hline $6-31+\mathrm{G}(\mathrm{d})$ & 1.0080 & 0.24 & 0.9772 & 0.25 & 1.0579 & 0.59 & 0.9024 & 0.28 \\
\hline $6-31+G(d, p)$ & 1.0099 & 0.24 & 0.9781 & 0.24 & 1.0334 & 0.38 & 0.9035 & 0.28 \\
\hline $6-31 G(2 d f, p)$ & 0.9946 & 0.22 & 0.9680 & 0.23 & 0.9858 & 0.23 & 0.8949 & 0.28 \\
\hline $6-311 \mathrm{G}(\mathrm{d})$ & 1.0123 & 0.24 & 0.9787 & 0.24 & 1.0143 & 0.39 & 0.9018 & 0.28 \\
\hline $6-311+\mathrm{G}(\mathrm{d})$ & 1.0174 & 0.24 & 0.9817 & 0.23 & 1.0437 & 0.52 & 0.9030 & 0.28 \\
\hline $6-311 G(d, p)$ & 1.0104 & 0.24 & 0.9781 & 0.23 & 0.9985 & 0.29 & 0.9021 & 0.28 \\
\hline $6-311+G(d, p)$ & 1.0161 & 0.24 & 0.9813 & 0.23 & 1.0158 & 0.32 & 0.9041 & 0.28 \\
\hline $6-311+G(2 d, p)$ & 1.0094 & 0.22 & 0.9778 & 0.21 & 1.0080 & 0.22 & 0.9044 & 0.27 \\
\hline $6-311+G(2 d f, p)$ & 0.9984 & 0.21 & 0.9709 & 0.21 & 0.9908 & 0.22 & 0.8959 & 0.28 \\
\hline $6-311+G(3 d f, p)$ & 0.9970 & 0.22 & 0.9689 & 0.22 & 0.9953 & 0.22 & 0.8953 & 0.29 \\
\hline $6-311+G(3 d f, 2 p)$ & 0.9963 & 0.22 & 0.9684 & 0.22 & 0.9952 & 0.22 & 0.8949 & 0.29 \\
\hline $6-311+\mathrm{G}(3 \mathrm{df}, 3 \mathrm{pd})$ & 0.9950 & 0.22 & 0.9679 & 0.22 & 0.9978 & 0.23 & 0.8938 & 0.29 \\
\hline $6-311++\mathrm{G}(3 \mathrm{df}, 3 \mathrm{pd})$ & 0.9950 & 0.22 & 0.9679 & 0.22 & 0.9983 & 0.23 & 0.8938 & 0.29 \\
\hline cc-pVDZ & 1.0093 & 0.25 & 0.9826 & 0.23 & 1.0042 & 0.26 & 0.9090 & 0.28 \\
\hline cc-pVTZ & 1.0010 & 0.21 & 0.9758 & 0.21 & 0.9960 & 0.21 & 0.8992 & 0.27 \\
\hline cc-pVQZ & 0.9997 & 0.20 & 0.9724 & 0.21 & $-e$ & $-e$ & 0.8978 & 0.27 \\
\hline aug-cc-pVDZ & $1.0339^{f}$ & $0.24^{f}$ & $1.0121^{f}$ & $0.31^{f}$ & 1.0452 & 0.34 & 0.9208 & 0.28 \\
\hline aug-cc-pVTZ & 1.0043 & 0.21 & 0.9778 & 0.22 & 1.0114 & 0.22 & 0.9009 & 0.28 \\
\hline aug-cc-pVQZ & 1.0010 & 0.20 & 0.9741 & 0.21 & $-e$ & $-^{e}$ & 0.8985 & 0.27 \\
\hline TZV2P & 1.0104 & 0.20 & 0.9798 & 0.20 & 1.0180 & 0.25 & 0.9054 & 0.26 \\
\hline
\end{tabular}

${ }^{a}$ Using the F1' set of 1062 frequencies, unless otherwise noted. ${ }^{b}$ Using the F1" set of 1033 frequencies. ${ }^{c}$ As defined by equation $14 .{ }^{d}$ Overall rootmean-square error in $\mathrm{S}_{\mathrm{vib}}(T)$ in units of $\mathrm{J} \mathrm{K}^{-1} \mathrm{~mol}^{-1}$. ${ }^{e}$ Scale factors and $\mathrm{rms}_{\mathrm{ov}}$ values not determined at these levels of theory. ${ }^{f} \mathrm{HCCCCH}$ has a bent structure at these levels of theory, and is therefore removed from the analysis. 
TABLE S13: Variation in Scale Factors and $\mathbf{r m s}_{\text {ov }}$ Values as a Function of the Percentage of Exact Exchange in Modified B3-LYP/6-31G(d) Procedures

\begin{tabular}{|c|c|c|c|c|c|c|}
\hline \multirow{2}{*}{$\begin{array}{c}\text { Percent exact } \\
\text { exchange }\end{array}$} & \multicolumn{2}{|c|}{$\mathrm{ZPVE}^{a}$} & \multicolumn{2}{|c|}{$\Delta H_{\mathrm{vib}}(298.15 \mathrm{~K})^{b}$} & \multicolumn{2}{|c|}{$S_{\mathrm{vib}}(298.15 \mathrm{~K})^{b}$} \\
\hline & scale factor ${ }^{c}$ & $\mathrm{rms}_{\mathrm{ov}}{ }^{d}$ & scale factor ${ }^{e}$ & $\mathrm{rms}_{\mathrm{ov}}{ }^{f}$ & scale factor ${ }^{g}$ & $\mathrm{rms}_{\mathrm{ov}}{ }^{h}$ \\
\hline 0 & 1.0485 & 0.045 & 1.0508 & 0.31 & 1.0127 & 0.54 \\
\hline 10 & 1.0228 & 0.039 & 1.0246 & 0.26 & 0.9967 & 0.46 \\
\hline 20 & 1.0004 & 0.035 & 1.0029 & 0.24 & 0.9813 & 0.42 \\
\hline 30 & 0.9804 & 0.034 & 0.9835 & 0.23 & 0.9669 & 0.40 \\
\hline 40 & 0.9623 & 0.034 & 0.9663 & 0.23 & 0.9532 & 0.40 \\
\hline 50 & 0.9458 & 0.035 & 0.9508 & 0.23 & 0.9399 & 0.42 \\
\hline 60 & 0.9308 & 0.036 & 0.9367 & 0.24 & 0.9274 & 0.45 \\
\hline 70 & 0.9169 & 0.038 & 0.9235 & 0.25 & 0.9153 & 0.48 \\
\hline 80 & 0.9044 & 0.041 & 0.9122 & 0.27 & 0.9036 & 0.51 \\
\hline 90 & 0.8932 & 0.044 & 0.9027 & 0.29 & 0.8923 & 0.54 \\
\hline 100 & 0.8834 & 0.049 & 0.8953 & 0.32 & 0.8814 & 0.57 \\
\hline
\end{tabular}

${ }^{a}$ Using the $\mathrm{Z} 1$ set of 89 frequencies. ${ }^{b}$ Using $\mathrm{F} 1$ ' set of 1062 frequencies. ${ }^{c}$ As defined by equation $8 .{ }^{d}$ Overall rms error in ZPVEs in $\mathrm{kJ} \mathrm{mol}^{-1}$. ${ }^{e}$ As defined by equation 13. ${ }^{f}$ Overall root-mean-square error in $\Delta H_{\mathrm{vib}}(T)$ in units of kJ $\mathrm{mol}^{-1}$. ${ }^{g}$ As defined by equation $14 .{ }^{h}$ Overall root-mean-square error in $\mathrm{S}_{\mathrm{vib}}(T)$ in units of $\mathrm{J} \mathrm{K}^{-1} \mathrm{~mol}^{-1}$. 
TABLE S14: Effect of Basis Set on ZPVE Scale Factors and Corresponding rms Values $^{a}$

\begin{tabular}{llllll}
\hline & \multicolumn{2}{c}{ CCSD } & & \multicolumn{2}{c}{$\operatorname{CCSD}(\mathrm{T})$} \\
\cline { 2 - 3 } \cline { 5 - 6 } Basis set & scale factor $^{b}$ & $\mathrm{rms}_{\mathrm{ov}}{ }^{b}$ & & scale factor & \\
\hline cc-pVDZ & 0.9823 & 0.26 & & 0.9913 & 0.43 \\
$\mathrm{rms}_{\mathrm{ov}}{ }^{b}$ \\
cc-pVTZ & 0.9773 & 0.18 & & 0.9878 & 0.28 \\
cc-pVQZ & $0.9758^{d}$ & $0.19^{d}$ & & $0.9867^{d}$ & $0.25^{d}$ \\
aug-cc-pVDZ & 0.9907 & 0.46 & & 1.0011 & 0.64 \\
aug-cc-pVTZ & 0.9790 & 0.17 & & 0.9900 & 0.28
\end{tabular}

${ }^{a}$ Using the Z1 set of 89 frequencies. ${ }^{b}$ As defined by equation $8 .{ }^{c}$ Overall rms error in ZPVEs in $\mathrm{kJ} \mathrm{mol}^{-1}$. ${ }^{d}$ Values obtained with $\mathrm{CH}_{2}=\mathrm{CH}_{2}$ removed from the analysis. 